\title{
A multi-omic cohort as a reference point for promoting a healthy human gut
}

\section{microbiome}

Authors: Zhuye Jie ${ }^{1,2,3^{*}, \dagger}$, Suisha Liang ${ }^{1, \dagger}$, Qiuxia Ding ${ }^{1, \dagger}, \mathrm{Fei} \mathrm{Li}^{1}$, Shanmei Tang ${ }^{1}$, Dan

Wang $^{1}$, Yuxiang $\operatorname{Lin}^{1}$, Peishan $\mathrm{Chen}^{1}$, Kaiye $\mathrm{Cai}^{1}$, Xuemei Qiu ${ }^{1}$, Qiang $\mathrm{Li}^{1}$, Yunli Liao ${ }^{1}$, Dongsheng Zhou ${ }^{1}$, Heng Lian ${ }^{1}$, Yong Zuo ${ }^{1}$, Xiaomin Chen ${ }^{1}$, Weiqiao Rao ${ }^{1}$, Yan Ren ${ }^{1}$, Yuan Wang ${ }^{1}$, Jin $\mathrm{Zi}^{1}$, Rong Wang ${ }^{1}$, Hongcheng Zhou ${ }^{4}$, Haorong $\mathrm{Lu}^{4}$, Xiaohan Wang ${ }^{1}$, Wei Zhang ${ }^{1}$, Tao Zhang ${ }^{1,3}$, Liang Xiao ${ }^{1,5,6}$, Yang Zong ${ }^{1}$, Weibin Liu ${ }^{1}$, Huanming Yang ${ }^{1,7}$, Jian Wang ${ }^{1,7}$, Yong Hou ${ }^{1}$, Xiao Liu ${ }^{1}$, Karsten Kristiansen ${ }^{1,3}$, Huanzi Zhong ${ }^{1,3}$, Huijue

$$
\mathrm{Jia}^{1,2,3,8, *}, \mathrm{Xun} \mathrm{Xu}^{1, *}
$$

\section{Affiliations:}

${ }^{1}$ BGI-Shenzhen, Shenzhen, China.

${ }^{2}$ Shenzhen Key Laboratory of Human Commensal Microorganisms and Health Research, BGI-Shenzhen, Shenzhen, China.

${ }^{3}$ Department of Biology, Ole MaalØes Vej 5, University of Copenhagen, Copenhagen, Denmark.

${ }^{4}$ China National Genebank, BGI-Shenzhen, Shenzhen 518120, China.

${ }^{5}$ Shenzhen Engineering Laboratory of Detection and Intervention of human intestinal microbiome, BGI-Shenzhen, Shenzhen, China.

${ }^{6}$ BGI-Qingdao, BGI-Shenzhen, Qingdao, 266555, China.

${ }^{7}$ James D. Watson Institute of Genome Sciences, Hangzhou, China.

${ }^{8}$ Macau University of Science and Technology, Avenida Wai long, Taipa, Macau, China. 
* To whom correspondence should be addressed. X.X. xuxun@genomics.cn ; Z.J.

jiezhuye@genomics.cn; H.J.jiahuijue@genomics.cn

$\uparrow$ These authors contributed equally to this work.

1 Abstract:

2 More than a decade of gut microbiome studies have a common goal for human

3 health. As most of the disease studies sample the elderly or the middle-aged, a

4 reference cohort for young individuals has been lacking. It is also not clear what

5 other omics data need to be measured to better understand the gut microbiome.

6 Here we present high-depth metagenomic shotgun sequencing data for the fecal

7 microbiome together with other omics data in a cohort of 2,183 adults, and observe

8 a number of vitamins, hormones, amino acids and trace elements to correlate with

9 the gut microbiome and cluster with T cell receptors. Associations with physical

10 fitness, sleeping habits and dairy consumption are identified in this large multi-omic

11 cohort. Many of the associations are validated in an additional cohort of 1,404

12 individuals. Our comprehensive data are poised to advise future study designs to

13 better understand and manage our gut microbiome both in population and in

14 mechanistic investigations.

16 The gut microbiome has been implicated in a growing list of complex diseases, showing

17 great potential for the diagnosis and treatment of metabolic, autoimmune and

18 neurological diseases as well as cancer. While case-control studies have been 
19 illuminating ${ }^{1}$, recently published studies have emphasized difficulty in extrapolating to

20 natural cohorts due to heterogeneity in location and ethnicity ${ }^{2,3}$. So far only a few cohorts

21 made use of metagenomic shotgun sequencing instead of 16S rRNA gene amplicon

22 sequencing, the largest being the LifeLines Deep cohort $(n=1,135,32$ million reads per

23 sample) from the Netherlands ${ }^{4-7}$. Fecal or plasma metabolites are more or less included

24 in gut microbiome studies, but the conclusions usually did not go beyond short-chain

25 fatty acids (SCFA), amino acids, vitamin B complex or bile acids. Levels of trace

26 elements such as arsenic have been a health concern (https://www.usgs.gov/mission-

27 areas/water-resources/science/arsenic-and-drinking-water?qt-

28 science_center_objects=0\#qt-science_center_objects,

29 https://www.fda.gov/food/metals/arsenic-food-and-dietary-supplements), but are

30 unexplored in the microbiome field. Biological sex is a strong determent for the gut

31 microbiome in mice and livestock ${ }^{8-10}$. The impact of hormones on the human gut

32 microbiome, or vice versa, remains unclear.

33 As part of the 4D-SZ (trans-omic, with more time points in future studies) cohort, here

34 we present metagenomic shotgun sequencing data sufficient for high-resolution

35 taxonomic and functional profiling $(86.1 \pm 23.3$ million reads per sample $)$ of the fecal

36 microbiome in a cohort of 2,183 adults, along with questionnaire data, physical fitness

37 tests, facial skin features, plasma metabolome and immune repertoire. Trans-omics

38 analyses in this Han Chinese cohort put into context fecal microbiome disease markers,

39 and uncover previously overlooked measurements such as aldosterone, testosterone, trace

40 elements and vitamin A that influence the gut microbiome, which were validated in an

41 additional cohort of 1,400 individuals. Trends for cardiometabolic diseases and colorectal 
42 cancer can be seen, despite the average age of 29.6. This is also to our knowledge the

43 largest cohort with facial skin data and immune repertoire data, which would also be of

44 interest for general health management and disease studies.

45 A recent study casted doubt over the health benefits of probiotic consumption, concluding

46 that colonization of the strains was highly variable between individuals ${ }^{11}$. Our large

47 cohort unequivocally showed commercial yogurt strains, especially Streptococcus

48 thermophilus and Bifidobacterium animalis in feces, and suggested beneficial effects in

49 cardiometabolic health.

\section{$51 \quad$ Results}

\section{Comprehensive gut microbiome data together with other omics}

53 Fecal samples were collected during a physical examination, and 2,183 samples (Age,

$5429.6 \pm 5.5$, average \pm stdev) were subjected to metagenomic shotgun sequencing, yielding

$5582.95 \pm 24.26$ million high-quality non-human reads per sample (Supplementary Table

56 1a), ensuring accurate taxonomic and functional profiling. The reads were mapped to a

57 comprehensive human gut microbiome reference gene catalog containing 9.9 million

58 genes (with a saturating mapping rate of $80.1 \pm 4.9 \%$ ) and then assigned to 1,507

59 Metagenomic Species (MGSs) ${ }^{12-14}$ and 2,981 metagenomic linkage groups (MLGs,

60 Kendall's tau instead of Pearson's or Spearman's correlation between genes) (Qin et al,

612012 , Jie et al, 2017), to include both known and unknown microbes.

62 Other omics data, including 104 plasma metabolites (3,980 samples), 634 immune

63 indices (PBMC (Peripheral blood mononuclear cells) V(D)J usage and its shannon 
64 diversity, 4,120 samples) from buffy coat, 72 basic medical data (body measurements and

65 routine blood test, 2,715 samples), 49 facial skin imaging indices (2,049 samples), 24

66 physical fitness data (3,833 samples), 18 entries from psychological questionnaire (2,039

67 samples), and 56 entries from lifestyle questionnaire (3,820 samples) were collected from

68 the same individuals (Fig. 1, Supplementary Tables 1b-d).

\section{The gut microbiome as a relatively independent dimension for health}

70 To get an overall idea of the relationship between omics, an inter-omics prediction value

71 between omics data was calculated using a 5-fold cross-validated random forest model

72 (RFCV, Fig. 2a). Basic medical data showed the highest global systematic association

73 with other omics data. The accuracy of prediction from basic medical data to physical

74 fitness data and from metabolites to basic medical data reaching $75 \%$ quantile showed

75 RFCV R = 0.461 and 0.399, respectively (Fig. 2a, b, Supplementary Fig. 1). Basic

76 medical data showed high prediction accuracy to metabolites (Fig. 2a, b); on the other

77 hand, serum creatinine, BMI, waist to hip ratio, hematocrit and triglyceride in basic

78 medical data can be predicted by metabolites (Fig. 2c). Metabolites constituted the

79 highest prediction accuracy to immune indices $(\mathrm{R}=0.292)(\mathrm{Fig} .2 \mathrm{~b})$. Immune indices

80 showed the second highest prediction accuracy to metabolites (Fig. 2a, b). Facial skin

81 features can be predicted by basic medical data, metabolites, physical fitness data and

82 lifestyle questionnaire (Fig. 2b, c). Among the lifestyle questionnaire, smoking, drinking

83 (especially low concentration alcohol), sports habits (especially resistance training), high-

84 sugar and high-fat dietary habit, and staying up until midnight can be predicted by other

85 omics data (Fig. 2c). 
86 A number of factors have been reported to explain gut microbial composition, while the

87 total percentage of variance explained remained in single digits ${ }^{4,15}$. According to a

88 RFCV predict model, we observe in this metagenomic cohort influence from lifestyle

89 questionnaire factors such as defecation, yogurt, age, gender, smoking, milk, soymilk,

90 drinking alcohol, fruit and vegetables on gut microbiome composition (Fig. 3), and the

91 cumulative effect size was also in single digits (Supplementary Tables 2b,2c). The BMI

92 distribution is narrow in this cohort (21.729 \pm 3.787 , Supplementary Table $1 b)$, so its

93 effect size was 0.0015 (q-value=0.014, Supplementary Table 2b). ABO blood group

94 could also predict fecal microbiome composition (RFCV R=0.2, Fig. 3), and specific

95 differences include Lachospiraceae bacterium 3_1_46FAA in blood type A $(q=6.12 \mathrm{E}-5)$,

96 Ruminococcus torques in blood type B (q = 1.59E-2), unnamed MGS209 in blood type

$97 \mathrm{AB}(\mathrm{q}=1.59 \mathrm{E}-2)$ and Megaspaera micronuciformis in blood type $\mathrm{O}(\mathrm{q}=1.69 \mathrm{E}-2)$.

98 As our 'other genome', the gut microbiome could predict other omics in this cohort. Gut

99 microbiome showed the greatest prediction power for metabolites, such as plasma

100 vitamins (vitamin A, folic acid, vitamin B5, vitamin D), plasma hormones (testosterone,

101 aldosterone), trace elements (mercury, selenium, arsenic) and plasma amino acids

102 (branched chain amino acids (BCAA), glutamic acid, tryptophan, tyrosine, histidine,

103 alanine) (Fig. 3). Interestingly, hand grip strength, vital capacity, speckles and pores on

104 cheeks and staying up until midnight can also be predicted by the gut microbiome (Fig. 3).

105 We next included a validation cohort of 1404 individuals (mean age 29.515 $55.248,480$

106 males and 570 females, $82.95 \pm 24.26$ million high-quality non-human reads per fecal

107 sample), which differed by hometown location compared to the initial cohort

108 (Supplementary Table 1a, Supplementary Table 1b, Fig. 1). The gut microbiome could 
109 also predict these plasma metabolites, with greater effects from mercury, cysteine,

110 selenium, iron and cobalt (Fig. 3, Supplementary Table 3), while other data such as

111 physical fitness tests and facial skin features are not available.

\section{Defecation, hormone and gender}

113 We see that gender (female 1,016, male 1,007) was one of the most significant factors to

114 diverge gut microbiome composition (Supplementary Fig.2a). Eubacterium dolichum,

115 and Blautia wexlerae were significantly more abundant in males (Supplementary Fig. 2a),

116 after adjusting for age, BMI, medication and dietary supplements (Supplementary Table

117 3b). Fusobacterium mortiferum, which positively associated with testosterone, was

118 sensitive to the statistical adjustments (Supplementary Tables 3a, 3b). Compared to males,

119 females showed a greater $\alpha$-diversity (Supplementary Table 2a, Supplementary Fig. 2c).

120 Bifidobacterium longum, B. bifidum, and B. catenulatum, B. pseudocatenulatum were all

121 significantly enriched in females, as well as potentially oral or vaginal bacteria such as

122 Streptococcus parasanguinis, Prevotella bivia (Supplementary Fig. 2a). Gut microbial

123 functional potential for secondary bile acids strongly associated with self-reported

124 defecation frequency, which were better validated than associations with sex hormones

125 (Supplementary Fig. 2b), suggesting that these are stable patterns.

126 Aldosterone, one of the major adrenal gland mineralcorticoid, positively correlated with

127 bacteria implicated in cardiometabolic health, such as Bacteroides intestinalis, $B$.

128 cellulosilyticus, B. stercorirosoris and Eubacterium eligens (Supplementary Fig. 3) ${ }^{16}$. E.

129 eligens and Ruminococcus lactaris scaled negatively with self-reported preference for a 
130 salty diet, in contrast to Blautia obeum (Supplementary Fig. 2a), and mice on a high salt

131 diet showed decrease in a number of commensal bacteria ${ }^{17}$.

\section{The metabolome-immune-gut axis}

133 Among the strongest associations between different omics is that between immune

134 repertoire and plasma metabolites (Fig. 2). More strikingly, when we plotted the

135 associations in detail, the clusters of metabolites corresponded either to the same TRBV

136 (T-cell receptor beta variable gene) or to the same TRBJ (T-cell receptor beta joining

137 gene) (Supplementary Fig. 4a). Vitamin A, 5-methyl four hydrogen folic acid, selenium,

138 mercury and serum aldosterone showed positive associations with a few TRBJ1-4 and

139 TRBJ2-1, and negative association with TRBJ2-4. Vitamin B5, Vitamin E, phosphoserine,

140 arginosuccinic acid and arsenic showed positive associations with TRBJ1-4, as well as

141 negative associations with TRBJ2-4, TRBV20-1 and TRBV3-1. Glutamic acid and serine

142 showed a pattern that were largely opposite to that of the vitamin A cluster, except for

143 negative associations with TRBV20-1.

144 We next explored how the gut microbiome might help put the metabolome-immune

145 associations into context. Vitamin A is central to a healthy immune system but is

146 typically studied for its role in early development ${ }^{18}$. A recent mice study reported

147 modulation of retinol dehydrogenase 7 expression and dampened antimicrobial response

148 in the gut by Clostridiale ${ }^{19}$. Consistently, we observed associations between Clostridia

149 species (Clostridia MGS0123, MGS0560, MGS0558, Lachnospiraceae bacterium

150 1_4_56FAA, Lachnospiraceae bacterium 6_1_63FAA, Lachnospiraceae bacterium

151 9_1_43BFAA, C, bolteae, Clostridium sp. AT4, Clostridium sp. M62.1) and vitamin A in

152 adult humans both with Spearman's corelation and with Masaslin associated 
153 (Supplementary Fig. 3, Supplementary Table 3a). 5-methyl four hydrogen folic acid

154 exhibited a positive correlation with Eubacterium eligens (Supplementary Fig. 4a,

155 Supplementary Fig. 3), a butyrate-producing bacterium that was relatively depleted in

156 atherosclerotic cardiovascular disease ${ }^{16} .5$-methyl four hydrogen folic acid also

157 negatively associated with Dorea and Blautia species (Supplementary Fig. 4a), which

158 have been implicated in obesity and could metabolize formate or hydrogen ${ }^{20-22}$

159 (Supplementary Fig. 4a). Associations between the gut microbiome and trace elements

160 including mercury, selenium and arsenic might be surprising (Supplementary Fig. 4a).

161 Selenium-containing rice is commercially promoted as anti-cancer, and we found that the

162 association pattern largely followed arsenic, consistent with these two trace elements'

163 similar function in anaerobic respiration ${ }^{23}$. Selenium and mercury also correlated with

164 disease-associated species such as Clostridium bolteae and Ruminococcus gnavus in the

165 gut microbiome.

166 The metabolome-immune cluster represented by phosphoserine, and argininosuccinic

167 acidnegatively associated with Bacteroides coprophilus (Supplementary Table 3i), a

168 prevalent but not very abundant species from the Bacteroides genus. MGSs from

169 Faecalibacterium prausnitzii (Supplementary Fig. 4a, Supplementary Fig. 3), a bacterium

170 reported to produce butyrate and metabolize arsenic ${ }^{24}$, positively associated with

171 L-homocitrulline, phosphoserine, negatively associated with vitamin A, mercury, as well

172 as with specific TCR V(D)J including positive correlation with TRBV27_TRBJ2.3 and

173 TRBV27_TRBJ2.5 and negative correlation with TRBV20-1:TRBJ2-4 (Supplementary

174 Fig. 4a, Supplementary Table 3). The third cluster represented by glutamic acid showed

175 negative associations with previously reported bacteria implicated with lower BMI such 
176 as Alistipes shahii, Bacteroides cellulosilyticus, Ruminococcus lactis and Eubacterium

177 eligens ${ }^{16}$ in this large cohort (Supplementary Fig. 4a), consistent with higher glutamic

178 acid in individuals with obesity or insulin resistance ${ }^{21,25}$, and here we tentatively

179 identified their associated TCRs (Supplementary Fig. 4a).

180 Moreover, gut microbiome functional potential showed specific associations with TCR

181 immune repertoire. The gut microbial module (GMM) ${ }^{26}$ for homoacetogenesis

182 (production of acetate from hydrogen and carbon dioxide) displayed widespread negative

183 associations, most notably with TRBV7-8:TRBJ2-2 (Supplementary Fig. 4b). TRBV7-8

184 frequency had been reported to be higher in Pima Indian individuals with Type 2 diabetes

$185{ }^{27}$ (Supplementary Table 3i). Modules for degradation of arginine and lysine, degradation

186 of lactose and galactose, also associated with a number of VJs (Supplementary Fig. 4b).

187 In the validation cohort, associations with fecal microbiome modules such as lysine

188 degradation, mucin degradation, lactose and galactose degradation, sulfate reduction were

189 validated (Supplementary Fig. 4b, Supplementary Table 3f), which was impressive given

190 the differences in trace metals and other metabolites between the two cohorts (Fig. 3,

191 Supplementary Table 1). So, from both taxonomic and functional points-of-view, the gut

192 microbiome is involved in the metabolome-immune interplay in circulation, with

193 important new leads for experimental investigations.

\section{Biomarkers for hyperuricemia and cardiometabolic diseases}

195 Hyperuricemia is common in the East Asian population, and urate is excreted in urine or

196 through the gastrointestinal tract. In our cohort, serum uric acid showed negative

197 correlations with gut bacteria such as Faecalibacterium prausnitzii, Alistipes shahii,

198 Oscillospiraceae and Bacteroides intestinalis (Fig. 4), adjusted for medication and 
199 dietary supplements. Moreover, serum uric acid positively correlated with vitamins

200 (vitamin A, B5, D3 and E), amino acids (glutamic acid and alanine), trace elements

201 (arsenic and mercury), while negatively associated with testosterone (Fig. 4). The

202 negative associations between fecal Butyricimonas virosa, Odoribacter splanchnicus and

203 plasma alanine were consistent with butyrate production from amino acids

204 (Supplementary Table $3 i)^{28,29}$, which together with methylhistidines hinted at a meat-

205 excess diet ${ }^{30}$. Self-reported dietary structure indeed showed association with serum uric

206 acid (Supplementary Table 3j). This is the first set of large-scale evidence for gut

207 microbiome dysbiosis in hyperuricemia, together with hormonal, metabolic and

208 potentially immunological differences.

209 We next defined a score according to 8 routine blood parameters and 80 fecal

210 microbiome features for cardiometabolic disease risk (see Methods) in this young cohort

211 and tested it in previously published case-control samples. With the fecal markers alone,

212 metagenomic samples from Chinese patients with atherosclerotic cardiovascular disease

213 (ACVD), liver cirrhosis, obesity and Crohn's disease all scored higher compared to

214 control samples without the disease $(\mathrm{P}<0.05)$ (Supplementary Fig. 5a), while those from

215 diseases such as colorectal cancer, rheumatoid arthritis and medication-unstratified T2D

216 did not (Supplementary Fig. 5a) ${ }^{16,21,31-35}$. The clinical parameters help clarified T2D and

217 Crohn's disease (Supplementary Fig. 5b). Thus, although regional differences and

218 misidentifications remain a concern, we illustrate the potential for population-wide

219 screens of cardiometabolic diseases using the fecal microbiome.

\section{Biomarkers for colorectal cancer}


221 This young multi-omic cohort also provide more insight into the relationship between gut

222 microbiome, plasma metabolome and colorectal cancer (CRC). Both the microbiome and

223 the plasma metabolome are being actively studied for CRC biomarkers, but to our

224 knowledge they have not been investigated in the same cohort. We see here that

225 previously reported CRC-enriched bacteria ${ }^{1,33,36,37}$ showed associations with plasma

226 metabolites regardless of statistical adjustment for covariates (Supplementary Table 3a).

227 Peptostreptococcus stomatis positively associated with plasma leucine, phenylalanine,

228 alanine, tyrosine, as well as sarcosine, a metabolite studied for prostate cancer and a

229 degradation intermediate of glycine betaine ${ }^{38,39}$ (Supplementary Fig. 6).

230 Enterobactericeae including Escherichia coli, Klebsiella pneumoniae, Enterobacter

231 cloacae and Citrobacter freundii positively associated with sarcosine, hydroxylysine,

232 branched chain amino acids, tyrosine, tryptophan, 1-methylhistidine, hydroxyproline,

233 and argininosuccinic acid (Supplementary Fig. 6). 1-methylhistidine is a marker for

234 habitual meat intake, especially red meat ${ }^{30}$. Bacteria such as Bacteroides

235 thetaiotaomicron, Butyricimonas virosa were more associated with 3-methylhistidine

236 (Supplementary Table 3a). Besides, the butyrate-producing E. eligens positively

237 associated with fruit and vegetable intake, while negatively associated with plasma

238 alanine (Fig. 4a, Supplementary Fig. 6). A number of these associations were also

239 observed in the validation cohort, e.g. Enterobacter cloacae and hydroxylysine, E.

240 eligens and alanine (Supplementary Table 3a). These results corroborate fecal markers of

241 CRC with plasma metabolites, and suggest further studies on the long-term interplay

242 between dietary metabolites and bacteria for CRC etiology and threshold for intervention.

\section{Physical fitness, exercising and sleeping reflected in the gut microbiome}


244 Vital capacity, a commonly used index to assess lung function, positively associated with

245 bacteria such as A. shahii, F. prausnitzii and Bifidobacterium adolescentis, while

246 negatively correlated with disease-related bacteria including Clostridium clostridioforme,

247 Ruminococcus gnavus and E. coli, regardless of statistical adjustments (Fig. 2, Fig. 5,

248 Supplementary Table 3e). Hand grip strength, a protective factor for cardiovascular

249 casualty ${ }^{40}$, negatively associated with E. coli (Fig. 5). Age and sex stratified vertical

250 jump score (Supplementary Table 4) negatively associated with E. coli, while positively

251 associated with B. cellulosilyticus, B. intestinalis, Eubacterium rectale, etc. Bacteroides

252 cellulosilyticus and B. stercorirosoris, which associated with exercise intensity, even

253 correlated with a faster reaction time (Fig. 5), reminding us with associations between $B$.

254 cellulosilyticus and aldosterone, B. stercorirosoris and folic acid in both cohorts

255 (Supplementary Table 3a). Moreover, gut microbiome diversity (Shannon index)

256 associated with favorable scores in most of the fitness tests (Supplementary Table 2a).

257 Besides, individuals who stay up until after midnight also showed negative correlations

258 with Holdemania filiformis, Veillonella atypica and 25-hydroxy vitamin D3/D, while

259 positively correlated with Clostridium hatheway, Clostridium phoceensis, mercury,

260 selenium, arsenic, vitamin A, hydroxyproline and phosphoserine (Supplementary Fig. 2a,

261 Supplementary Fig. 5, Supplementary Table 3b). Thus, sleeping is also a factor to

262 consider for a complete understanding of the gut microbiome.

\section{Species from yogurt in the healthy gut microbiome}

264 Besides defecation frequency and gender, yogurt consumption explained a notable

265 portion of variances in the gut microbiome (Fig. 3, Supplementary Table 2b). A recent

266 study casted doubt over the health benefits of probiotics, concluding that colonization of 
267 the bacteria was highly variable between individuals ${ }^{11}$. In both our large cohorts,

268 Streptococcus thermophilus, a species included in commercial yogurt mainly for its

269 thermal stability and metabolic support for other strains, was consistently detected in

270 yogurt eaters, and scaled with self-reported frequency of yogurt consumption (Fig. 6,

271 Supplementary Fig. 7). Bifidobacterium animalis, likely representing the star strain from

272 CHR HANSEN, B. animalis subsp. lactis BB-12, was also enriched in yogurt eaters, and

273 fecal relative abundance of $B$. animalis associated with less stress, less bilirubin, lower

274 diastolic blood pressure, as well as with TCR V(D)J combinations (Fig. 6d), suggesting

275 immune modulation. The association between $B$. animalis and TRBV5.6:TRBJ2.5 was

276 also observed in the validation cohort (Supplementary Table 3c), while the other

277 parameters were unfortunately not available. In contrast to $S$. thermophilus, B. animalis,

278 and Veillonella, there was no significant increase in any Lactobacillus strains (Fig. 6).

279 Those who used to take yogurt also showed less Clostridium bolteae, a bacterium known

280 to be elevated in a number of cardiometabolic diseases ${ }^{1,16}$. Intriguingly, fecal C. bolteae

281 associated with plasma triglyceride, uric acid, phosphoserine, vitamin A, and mercury

282 (Fig. 6e), offering an explanation for epidemiological evidence of yogurt consumption

283 and reduced risk of gout ${ }^{41}$. In the validation cohort, C. bolteae also associated with

284 mercury and to a lesser extent vitamin A (the vitamin A association was sensitive to

285 covariates, Supplementary Table 3a). Besides, yogurt consumption was associated with a

286 number of favorable measurements such as higher HDL (high-density lipoprotein)

287 cholesterols, lower uric acid and triglycerides, less cysteine, mercury and hydroxyproline 288 (Fig. 6a). 
289 Regarding Bifidobacterium in the gut microbiome, however, individuals who consumed

290 milk enriched for B. longum, B. catenulatum and B. pseudocatenulatum (Fig. 6a, b),

291 implying that some of the yogurt-associated differences come from its exogenous strains

292 such as $S$. thermophilus and B. animalis, as well as less $C$. bolteae. The higher

293 Bifidobacterium spp., and lower Blautia wexlerae and Ruminococcus sp. 5_1_39BFAA

294 associated with milk intake were validated in the additional 1404 individuals

295 (Supplementary Table 3). Milk drinking also associated with vitamin B2, B5, B6, HDL,

296 lymphocytes, etc. in the blood, vital capacity, and psychological scores (Fig. 6a, b,

297 Supplementary Fig. 7).

\section{Discussion}

\section{Insights from multi-omics}

301 In summary, our trans-omic investigation of thousands volunteers establish an

302 unprecedented reference data set for the human gut microbiome. Judging from the

303 associations, it appears as though a number of factors in circulation crosstalk with the gut

304 microbiome, and then manifest on the face, in the head and in fitness tests. Levels of trace

305 elements, such as mercury, arsenic and selenium, as important cofactors for bacteria

306 respiration and other functions ${ }^{23}$, should be measured even in uncontaminated regions,

307 and in individuals showing normal levels of these elements. Although rice is often

308 studied for such contaminants, exposure can be from other food, drink, air and soil

309 sources $^{42}$ (https://www.fda.gov/food/metals/arsenic-food-and-dietary-supplements). Our

310 results suggest that commensal microbial metabolism of trace elements might help

311 determine their levels in the blood, and influence immune functions. 
312 The PBMC TCR $\beta$ CDR3 V(D)J usage in such a large cohort is a great resource for

313 discovering microbial antigens other than those from traditional pathogens. While some

$314 T R B V$ and TRBJ segments are more frequent than others ${ }^{43}$, we do not yet know how they

315 correspond to T cell sub-populations. Existing studies on TCR have been focusing on

316 pathogens, autoimmune diseases and cancer. For example, TCR profiles of tumor-

317 resident $\mathrm{T}_{\text {reg }}$ (regulatory $\mathrm{T}$ ) cells have been shown to significantly overlap with those of

318 circulating $\mathrm{T}_{\text {reg }}$ cells ${ }^{44}$; immune phenotype of peripheral blood $\mathrm{T}_{\text {reg }}$ II cells was not only

319 similar to that of intratumoral $\mathrm{T}_{\text {reg }}$ cells, but also predicted future relapse of breast cancer

320 patients ${ }^{45}$. A high diversity in the $\mathrm{T}$ cell immune repertoire is believed to be preferable,

321 but the $\mathrm{T}$ cell immune repertoire diversity has been reported to be unchanged after a 3-

322 month switch from omnivorous to vegetarian and lower in long-term vegetarians ${ }^{46}$. In

323 our analyses of this cohort, the overall diversity (Immunity index, Methods) was not the

324 most important factor that predicted other omics, yet could be reflected by metabolites,

325 physical fitness tests, lifestyle and skin features (RFCV R 0.2, Fig. 2,3). We identify

326 clustering patterns of specific TCR $\beta$ CDR3 VJ joining with plasma metabolites including

327 vitamins, trace elements and amino acids (Supplementary Fig. 4a). The chains of

328 causality remain to be fully elucidated; yet, it is likely to be a two-way interplay for

329 metabolite-gut microbiome, metabolite-T cells, and gut microbiome-T cells. Our results

330 imply long-term differences in these features in apparently healthy individuals. A similar

331 speculation could be made for facial skin features, which we expect to be resilient against

332 topical interventions judging from the strong associations with blood parameters.

333 We have tentatively identified gut bacteria associated with each ABO blood type. A

334 larger proportion of blood type A in Europeans compared to East Asians might help 
335 explain the greater abundance of Lachospiraceae bacterium ${ }^{12,47}$. Blood type B is more

336 prevalent in northern Chinese, and the blood type B-enriched mucin-degrading bacterium

$337 R$. torques has recently been reported to show an association with blood glucose ${ }^{48}$ and

338 was also associated with ulcerative colitis ${ }^{49}$ and a Bristol stool score of 1 or 2

339 (Supplementary Fig. 2a). Megaspaera micronuciformis, seen in association with blood

340 type $\mathrm{O}$, can produce butyrate from acetate ${ }^{50}$. Genetic studies of the gut microbiota have

341 not yet reported genome-wide significant associations with ABO blood type genes

342 themselves ${ }^{51-54}$, while multiple studies have reported impact of FUT2 secretor/non-

343 secretor status on gut microbiota composition ${ }^{55-57}$. Tentative associations here are yet to

344 be matched with in vitro studies with the glycans ${ }^{58,59}$.

345 Differences in gut microbiome composition between sexes and a greater microbial

346 diversity in females have recently been reported in the LifeLines Deep cohort, yet the gut

347 microbiome in females was influenced by oral contraceptives, ovariectomy as well as

348 antibiotics for vaginal or pelvic infections ${ }^{60}$. Males of Hadza hunter-gatherers showed

349 differences in gut microbiota compared to females ${ }^{61}$, including higher Eubacterium

350 and Blautia in men which were also recapitulated in our Chinese cohort (E. dolichum, B.

351 wexlerae). Interestingly, E. dolichum associated with a dietary structure of more meat

352 instead of fruit and vegetables, while $B$. wexlerae scaled negatively with milk

353 consumption (Supplementary Fig. 2, Fig. 6). The evolutionary implications remain

354 unclear.

355 A baseline of the gut microbiome with deviations towards diseases

356 Metagenome-wide association studies (MWAS) have documented gut microbial

357 perturbations in a growing list of diseases by comparing cases versus controls. Here we 
358 provide a high-depth metagenomic cohort, the mean age for which did not exceed 30

359 years old. Alarmingly enough, trends for cardiometabolic diseases and colorectal cancer

360 can already been seen from the fecal microbiome and a few parameters in the blood. The

361 set of healthy gut microbes for leanness are increasingly clear ${ }^{16}$, such as A. shahii, F.

362 prausnitzii, E. eligens and B. cellulosilyticus. And we have a better idea how to increase

363 their relative abundances. Interestingly, we observed few association with Akkermansia,

364 which may indeed be too diverse among individuals ${ }^{5,62}$ or require mucosal sampling. The

365 list of potentially harmful gut microbes are also increasingly clear; future studies are

366 needed to confirm whether we can decrease E. coli and $R$. gnavus with exercising and

367 diet, fend off $C$. boltae with yogurt, etc.

368 While an older cohort would be needed to look at type 2 diabetes ${ }^{63,64}$, hyperuricemia is

369 common in this cohort (Supplementary Table 1). A. shahii negatively associated with

370 plasma tryptophan (Fig. 4, Supplementary Table 3i), and hyperuricemia has been

371 reported to skew tryptophan metabolism towards kynurenine production in mice models

$372{ }^{65}$, instead of indole reported for $A$. shahii ${ }^{66}$, potentially modulating signaling through

373 aryl hydrocarbon receptors $(\mathrm{AhR})^{67}$. One of the bacteria negatively associated with

374 serum uric acid, $F$. prausnitzii, has been reported to encode a methyltransferase for

375 arsenic detoxification (Supplementary Table $3 i$ ) ${ }^{24}$. IL-1 $\beta$, the major cytokine responsible

376 for gout ${ }^{68}$, has been associated with urinary level of arsenic ${ }^{69}$. Co-stimulation of patient-

377 derived PBMCs with monosodiurm urate crystals and TLR2 or TLR4 (toll-like receptors)

378 ligands have been shown to disrupt IL-1 $\beta /$ IL-1Ra (IL1 receptor antagonist) balance ${ }^{70}$,

379 consistent with involvement of microbes in gout. 
380 Genetic potential for histidine degradation instead of synthesis have been observed to

381 increase in CRC relative to healthy controls according to metagenomic studies ${ }^{37,71} .1$ -

382 methylhistidine, a marker for habitual meat intake ${ }^{30}$, could be metabolized into histidine.

383 Plasma level of the amino acid proline was reported to increase in a mouse model of

$384 \mathrm{CRC}^{72}$, but found in another study to decrease in human $\mathrm{CRC}^{73}$. In this young cohort

385 from China, we did not see significant associations between proline and known gut

386 microbiome markers of CRC. Hydroxyproline, on the other hand, is better predicted by

387 the gut microbiome composition compared to proline (Fig. 3), and associated with meat

388 consumption, staying up until after 0 am (Supplementary Fig. 7). Enterobacteriaceae such

389 as Escherichia coli and Klebsiella pneumoniae positively associated with hydroxyproline

390 in this cohort. A recent study analyzed fecal metabolites together with fecal microbiome

391 and reported among others an increase in branched chain amino acids and aromatic amino

392 acids in $\mathrm{CRC}^{74}$. Here we observe plasma levels of these amino acids to associate with

393 CRC markers such as P. stomatis, and E. coli, while the fecal metagenomic potential for

394 leucine biosynthesis was control-enriched ${ }^{37,74}$, implying that leucine was normally not in

395 excess.

396 We also find it intriguing that decarboxylases appear generally important for bacterial

397 stress response in the microbiome, i.e. to maintain a balanced $\mathrm{pH}$ for themselves. The top

398 one for gut microbes may be glutamate decarboxylase (produces GABA ( $\gamma$-aminobutyric

399 acid) from glutamate), while histidine decarboxylase in the female reproductive tract

400 might contribute to menstrual pains ${ }^{75}$. Besides, recent studies identified tyrosine

401 decarboxylases in gut microbes that could digest the medication levodopa used to treat

402 Parkinson's disease ${ }^{76,77}$. 


\section{Behavioral changes to be trialed for a healthy gut microbiome?}

404 Although effects of sleep fragmentation on hemopoiesis have been seen despite antibiotic

405 treatment ${ }^{78}$, our results nonetheless suggest that the gut microbiome may have an

406 additional role, together with trace elements, vitamins, and host genetics ${ }^{79}$. The less

407 hypocretin in mice subjected to sleep fragmentation promoted atherosclerosis ${ }^{78}$. The

408 increased adiposity and decreased lean mass with sleep loss also involved toll-like

409 receptors (TLRs) ${ }^{80,81}$, and we identify cardiometabolic disease-associated species

410 including Clostridium hatheway here.

411 Potential influence of physical activity on the gut microbiota has been analyzed in small

412 cohorts of rugby athletes ${ }^{82}$ and colorectal cancer ${ }^{37}$. Although more detailed information

413 for physical activity is preferable, compliance to recordings such as Fitbit is notoriously

414 bad in healthy individuals ${ }^{83}$. Results from this large cohort at least suggest that

415 exercising might help improve cardio-pulmonary function (grip strength, vital capacity)

416 to decrease incidence of cardiometabolic diseases. Intense exercise, explored for

417 application to individuals with diseases such as prediabetics and Alzheimer's ${ }^{84,85}$, may be

418 no less important than endurance or resistance training; and our results suggest that

419 different types of exercise could have differential impacts on the gut microbiome and the

420 microbiome changes could be a readout for monitoring effects of training. Endurance

421 training actually lowers testosterone ${ }^{86}$ and could lead to hyperuricemia, especially if

422 combined with high-fructose food and drinks and lack of dairy consumption ${ }^{41}$.

423 Our large-scale analyses provide substantial support for health benefits of yogurt

424 consumption. The universally present species were Streptococcus thermophilus and

425 Bifidobacterium animalis instead of commonly tested probiotics from Lactobacillus. An 
426 orally administered strain of B. longum has been shown to persist in $30 \%$ of individuals

427 for at least 6 months ${ }^{87}$, while we failed to detect in feces an L. casei strain gavaged to

428 rats ${ }^{88,89}$, suggesting general differences between Bifidobacterium and Lactobacillus. The

429 strains used by Zmora et al. included a number of Lactobacillus, Bifidobacterium, as well

430 as Streptococcus and Lactococcus, all detectable in various gastrointestinal sites despite

431 laxative and colonoscopy ${ }^{11}$. One potential explanation for the association with desirable

432 cardiometabolic and psychological scores observed in our study for yogurt or milk is the

433 production of metabolites such as folate and GABA by S. thermophilus, Bifidobacterium

434 and Lactobacillus ${ }^{90,91}$. Moreover, Lactobacilli have been reported to sequester heavy

435 metals including lead and cadmium ${ }^{92}$. All of these live or dead probiotics could

436 potentially exert functions on the immune system or even the brain. The positive

437 association with endogenous Bifidobacterium species with milk intake is more likely due

438 to live bacteria which help metabolize the lactose in this largely lactose-intolerant

439 population. It remains to be seen whether and how diary consumption affects the gut

440 microbiome in other cohorts, and there appears to be regional differences in China

441 already.

442 Thus, this study provides a young reference for the gut microbiome with physical fitness

443 test and questionnaire data, and reveals interrelationship with other omics such as trace

444 elements, hormones and immune repertoire that have so far not been included in other

445 study designs. There is a lot more to investigate both in vitro and in vivo by researchers

446 across disciplines. Interventional as well as mechanistic studies will be needed to see how

447 physical activity, well-timed sleeping and dietary interventions such as yogurt, milk and 
448 vegetables might improve the gut microbiome, hormone levels, cardiometabolic and

449 mental health.

451 Data and materials availability: Metagenomic sequencing data for all samples have

452 been deposited to the CNSA (https://db.cngb.org/cnsa/) of (CNGB) database under the

453 accession code CNP00 00426, CNP0000289.

\section{Acknowledgments}

456 The authors are very grateful to colleagues at BGI-Shenzhen and China National

457 Genebank (CNGB), Shenzhen for sample collection, DNA extraction, library

458 construction, sequencing, and discussions. We thank Dr. Qiang Sun (University of

459 Toronto), our colleagues Chen Chen and Yanmei Ju, Jinghua Wu for helpful comments.

\section{Author contributions:}

462 J.W. initiated the overall health project. Y.Z., H.Z., K.C., P.C., X.X. organized the

463 sample collection and processing, with immune repertoire from X.L., W.Z, metabolomics

464 from X.Q., Q.L., Y.L., D.Z., H.Lian, Y.Z., X.C., W.R., Y.R., Y.W., J.Z., R.W., raw

465 metagenomic profile from Q.D, X.W., and J.Z., Q.D., S.T., Y.L., D.W. checked the host

466 metadata and matched the omics data. H.Zhou, H.Lu led the DNA extraction and

467 sequencing, respectively. Z.J. led the bioinformatic analyses, including S.L. and F.L.

468 H.Zhong, Q.D., S.T., D.W. performed early analyses which are not included in the 

rendered the display items. All authors contributed to finalizing this manuscript.

472 Competing interests: The authors declare no competing financial interest.

475 1. Wang, J. \& Jia, H. Metagenome-wide association studies: fine-mining the 476 microbiome. Nat. Rev. Microbiol. 14, 508-522 (2016).

4772 . He, Y. et al. Regional variation limits applications of healthy gut microbiome 478 reference ranges and disease models. Nat. Med. 24, 1532-1535 (2018).

4793 . Deschasaux, M. et al. Depicting the composition of gut microbiota in a population 480 with varied ethnic origins but shared geography. Nat. Med. (2018) doi:10.1038/s41591481 018-0160-1.

482 4. Zhernakova, A. et al. Population-based metagenomics analysis reveals markers 483 for gut microbiome composition and diversity. Science 352, 565-569 (2016).

484 5. Xie, H. et al. Shotgun Metagenomics of 250 Adult Twins Reveals Genetic and 485 Environmental Impacts on the Gut Microbiome. Cell Syst. 3, 572-584.e3 (2016). 6. Rothschild, D. et al. Environment dominates over host genetics in shaping human gut microbiota. Nature 555, 210-215 (2018).

7. Mehta, R. S. et al. Stability of the human faecal microbiome in a cohort of adult men. Nat. Microbiol. 3, 347-355 (2018).

8. Xiao, L. et al. A catalog of the mouse gut metagenome. Nat. Biotechnol. 33, 1103-1108 (2015).

9. Xiao, L. et al. A reference gene catalogue of the pig gut microbiome. Nat.

494 10. Zhao, L. et al. Quantitative genetic background of the host influences gut 495 microbiomes in chickens. Sci. Rep. 3, 1163 (2013).

496 11. Zmora, N. et al. Personalized Gut Mucosal Colonization Resistance to Empiric

497 Probiotics Is Associated with Unique Host and Microbiome Features. Cell 174, 1388-

498 1405.e21 (2018).

499 12. Li, J. et al. An integrated catalog of reference genes in the human gut microbiome. 500 Nat. Biotechnol. 32, 834-841 (2014).

501 13. Nielsen, H. B. et al. Identification and assembly of genomes and genetic elements 502 in complex metagenomic samples without using reference genomes. Nat. Biotechnol. 32 , 503 822-828 (2014).

504 14. Moll1, J. M. et al. Pathway-based approach to classify unannotated gut bacteria 505 associated with insulin sensitivity. Revis. Nat Microbiol (2019). 
506 15. Falony, G. et al. Population-level analysis of gut microbiome variation. Science 507 352, 560-564 (2016).

508 16. Jie, Z. et al. The gut microbiome in atherosclerotic cardiovascular disease. Nat. 509 Commun. 8, 845 (2017).

510 17. Wilck, N. et al. Salt-responsive gut commensal modulates TH17 axis and disease.

$511 \quad$ Nature 551, 585-589 (2017).

512 18. Veldhoen, M. \& Ferreira, C. Influence of nutrient-derived metabolites on

513 lymphocyte immunity. Nat. Med. 21, 709-718 (2015).

514 19. Grizotte-Lake, M. et al. Commensals Suppress Intestinal Epithelial Cell Retinoic

515 Acid Synthesis to Regulate Interleukin-22 Activity and Prevent Microbial Dysbiosis.

516 Immunity 49, 1103-1115.e6 (2018).

517 20. Lawson, P. A. et al. Reclassification of Eubacterium formicigenerans Holdeman

518 andMoore 1974 as Dorea formicigenerans gen. nov., comb. nov., and description of

519 Dorea longicatena sp. nov., isolated from human faeces. Int. J. Syst. Evol. Microbiol. 52,

520 423-428 (2002).

521 21. Liu, R. et al. Gut microbiome and serum metabolome alterations in obesity and

522 after weight-loss intervention. Nat. Med. 23, 859-868 (2017).

523 22. Beaumont, M. et al. Heritable components of the human fecal microbiome are

524 associated with visceral fat. Genome Biol. 17, 189 (2016).

525 23. Stolz, J. F. \& Oremland, R. S. Bacterial respiration of arsenic and selenium.

526 FEMS Microbiol. Rev. 23, 615-27 (1999).

527 24. Coryell, M., McAlpine, M., Pinkham, N. V., McDermott, T. R. \& Walk, S. T. The

528 gut microbiome is required for full protection against acute arsenic toxicity in mouse

529 models. Nat. Commun. 9, 5424 (2018).

530 25. Cheng, S. et al. Metabolite profiling identifies pathways associated with

531 metabolic risk in humans. Circulation 125, 2222-31 (2012).

532 26. Vieira-Silva, S. et al. Species-function relationships shape ecological properties

533 of the human gut microbiome. Nat. Microbiol. 29, 16088 (2016).

534 27. Frankl, J. A., Thearle, M. S., Desmarais, C., Bogardus, C. \& Krakoff, J. T-cell

535 receptor repertoire variation may be associated with type 2 diabetes mellitus in humans.

536 Diabetes Metab. Res. Rev. 32, 297-307 (2016).

537 28. Sakamoto, M. et al. Butyricimonas synergistica gen. nov., sp. nov. and

538 Butyricimonas virosa sp. nov., butyric acid-producing bacteria in the family

539 'Porphyromonadaceae' isolated from rat faeces. Int. J. Syst. Evol. Microbiol. 59, 1748-

5401753 (2009).

$54129 . \quad$ Vital, M., Howe, A. C. \& Tiedje, J. M. Revealing the bacterial butyrate synthesis

542 pathways by analyzing (meta)genomic data. mBio 5, e00889 (2014).

543 30. Mitry, P. et al. Plasma concentrations of anserine, carnosine and pi-

544 methylhistidine as biomarkers of habitual meat consumption. Eur. J. Clin. Nutr. 73, 692-

545702 (2019).

546 31. He, Q. et al. Two distinct metacommunities characterize the gut microbiota in

547 Crohn's disease patients. GigaScience 6, 1-11 (2017).

548 32. Qin, N. et al. Alterations of the human gut microbiome in liver cirrhosis. Nature

549 513, 59-64 (2014).

550 33. Yu, J. et al. Metagenomic analysis of faecal microbiome as a tool towards

551 targeted non-invasive biomarkers for colorectal cancer. Gut 66, 70-78 (2017). 
552 34. Zhang, X. et al. The oral and gut microbiomes are perturbed in rheumatoid arthritis and partly normalized after treatment. Nat. Med. 21, 895-905 (2015).

554 35. Qin, J. et al. A metagenome-wide association study of gut microbiota in type 2

555 diabetes. Nature 490, 55-60 (2012).

556 36. Zeller, G. et al. Potential of fecal microbiota for early-stage detection of colorectal 557 cancer. Mol. Syst. Biol. 10, 766 (2014).

558 37. Feng, Q. et al. Gut microbiome development along the colorectal adenoma559 carcinoma sequence. Nat. Commun. 6, 6528 (2015).

560 38. Sreekumar, A. et al. Metabolomic profiles delineate potential role for sarcosine in 561 prostate cancer progression. Nature 457, 910-914 (2009).

562 39. Hai, Y., Huang, A. M. \& Tang, Y. Structure-guided function discovery of an

563 NRPS-like glycine betaine reductase for choline biosynthesis in fungi. Proc. Natl. Acad.

564 Sci. 116, 10348-10353 (2019).

565 40. Tikkanen, E., Gustafsson, S. \& Ingelsson, E. Associations of Fitness, Physical

566

567 Activity, Strength, and Genetic Risk With Cardiovascular Disease: Longitudinal

568

569

570

571 Analyses in the UK Biobank Study. Circulation 137, 2583-2591 (2018).

41. Kuo, C.-F., Grainge, M. J., Zhang, W. \& Doherty, M. Global epidemiology of gout: prevalence, incidence and risk factors. Nat. Rev. Rheumatol. 11, 649-662 (2015). 42. Chen, L. et al. Trans-provincial health impacts of atmospheric mercury emissions in China. Nat. Commun. 10, 1484 (2019).

572 43. Freeman, J. D., Warren, R. L., Webb, J. R., Nelson, B. H. \& Holt, R. A. Profiling 573 the T-cell receptor beta-chain repertoire by massively parallel sequencing. Genome Res.

574 19, 1817-1824 (2009).

575 44. Ahmadzadeh, M. et al. Tumor-infiltrating human CD4 + regulatory T cells

576 display a distinct TCR repertoire and exhibit tumor and neoantigen reactivity. Sci.

577 Immunol. 4, eaao4310 (2019).

578 45. Wang, L. et al. Connecting blood and intratumoral Treg cell activity in predicting 579 future relapse in breast cancer. Nat. Immunol. (2019) doi:10.1038/s41590-019-0429-7.

580 46. Zhang, C. et al. Impact of a 3-Months Vegetarian Diet on the Gut Microbiota and 581 Immune Repertoire. Front. Immunol. 9, (2018).

582 47. Davenport, E. R. et al. ABO antigen and secretor statuses are not associated with 583 gut microbiota composition in 1,500 twins. BMC Genomics 17, 941 (2016).

584 48. Zeevi, D. et al. Structural variation in the gut microbiome associates with host 585 health. Nature (2019) doi:10.1038/s41586-019-1065-y.

586 49. Png, C. W. et al. Mucolytic bacteria with increased prevalence in IBD mucosa 587 augment in vitro utilization of mucin by other bacteria. Am. J. Gastroenterol. 105, 2420$588 \quad 8$ (2010).

589 50. Marchandin, H. et al. Phylogenetic analysis of some Sporomusa sub-branch

590 members isolated from human clinical specimens: description of Megasphaera

591 micronuciformis sp. nov. Int. J. Syst. Evol. Microbiol. 53, 547-53 (2003).

592 51. Wang, J. et al. Genome-wide association analysis identifies variation in vitamin D 593 receptor and other host factors influencing the gut microbiota. Nat. Genet. 48, 1396-1406 594 (2016).

595 52. Bonder, M. J. et al. The effect of host genetics on the gut microbiome. Nat. Genet. 596 48, 1407-1412 (2016). 
597

598

599

600

601

602

603

604

605

606

607

608

609

610

611

612

613

614

615

616

617

618

619

620

621

622

623

624

625

626

627

628

629

630

631

632

633

634

635

636

637

638

639

640

641

53. Turpin, W. et al. Association of host genome with intestinal microbial composition in a large healthy cohort. Nat. Genet. (2016) doi:10.1038/ng.3693.

54. Liu, X. et al. M-GWAS for the Gut Microbiome in Chinese Adults Illuminates on Complex Diseases. Cell (2019) doi:10.2139/ssrn3383800.

55. Rausch, P. et al. Colonic mucosa-associated microbiota is influenced by an interaction of Crohn disease and FUT2 (Secretor) genotype. Proc. Natl. Acad. Sci. 108, 19030-19035 (2011).

56. Rausch, P. et al. Multigenerational Influences of the Fut2 Gene on the Dynamics of the Gut Microbiota in Mice. Front. Microbiol. 8, 991 (2017).

57. Zhernakova, D. V. et al. Individual variations in cardiovascular-disease-related protein levels are driven by genetics and gut microbiome. Nat. Genet. (2018) doi:10.1038/s41588-018-0224-7.

58. Liu, Q. P. et al. Bacterial glycosidases for the production of universal red blood cells. Nat. Biotechnol. 25, 454-464 (2007).

59. Rahfeld, P. et al. An enzymatic pathway in the human gut microbiome that converts A to universal O type blood. Nat. Microbiol. (2019) doi:10.1038/s41564-0190469-7.

60. Sinha, T. et al. Analysis of 1135 gut metagenomes identifies sex-specific resistome profiles. Gut Microbes 1-9 (2018) doi:10.1080/19490976.2018.1528822.

61. Schnorr, S. L. et al. Gut microbiome of the Hadza hunter-gatherers. Nat. Commun. 5, 3654 (2014).

62. Schloissnig, S. et al. Genomic variation landscape of the human gut microbiome. Nature 493, 45-50 (2012).

63. Schüssler-Fiorenza Rose, S. M. et al. A longitudinal big data approach for precision health. Nat. Med. 25, 792-804 (2019).

64. Zhou, W. et al. Longitudinal multi-omics of host-microbe dynamics in prediabetes. Nature 569, 663-671 (2019).

65. Dankers, A. C. A. et al. Hyperuricemia influences tryptophan metabolism via inhibition of multidrug resistance protein 4 (MRP4) and breast cancer resistance protein (BCRP). Biochim. Biophys. Acta 1832, 1715-22 (2013).

66. Song, Y. et al. Alistipes onderdonkii sp. nov. and Alistipes shahii sp. nov., of human origin. Int. J. Syst. Evol. Microbiol. 56, 1985-90 (2006).

67. Cervenka, I., Agudelo, L. Z. \& Ruas, J. L. Kynurenines: Tryptophan's metabolites in exercise, inflammation, and mental health. Science 357, eaaf9794 (2017).

68. So, A. K. \& Martinon, F. Inflammation in gout: mechanisms and therapeutic targets. Nat. Rev. Rheumatol. (2017) doi:10.1038/nrrheum.2017.155.

69. Parvez, F. et al. Assessment of arsenic and polycyclic aromatic hydrocarbon (PAH) exposures on immune function among males in Bangladesh. PloS One 14, e0216662 (2019).

70. Cri $\square$ an, T. O. et al. Soluble uric acid primes TLR-induced proinflammatory cytokine production by human primary cells via inhibition of IL-1Ra. Ann. Rheum. Dis. 75, 755-62 (2016).

71. Thomas, A. M. et al. Metagenomic analysis of colorectal cancer datasets identifies cross-cohort microbial diagnostic signatures and a link with choline degradation. Nat. Med. 25, 667-678 (2019). 
642 72. Manna, S. K. et al. Biomarkers of Coordinate Metabolic Reprogramming in 643 Colorectal Tumors in Mice and Humans. Gastroenterology 146, 1313-1324 (2014).

644 73. Qiu, Y. et al. Serum metabolite profiling of human colorectal cancer using GC-

645 TOFMS and UPLC-QTOFMS. J Proteome Res 8, 4844-4850 (2009).

646 74. Yachida, S. et al. Metagenomic and metabolomic analyses reveal distinct stage-

647 specific phenotypes of the gut microbiota in colorectal cancer. Nat. Med. (2019)

648 doi:10.1038/s41591-019-0458-7.

$64975 . \quad J i e$, Z. et al. The vagino-cervical microbiome as a woman's life history. Revis.

650 Cell (2019) doi:10.1101/533588.

651 76. van Kessel, S. P. et al. Gut bacterial tyrosine decarboxylases restrict levels of

652 levodopa in the treatment of Parkinson's disease. Nat. Commun. 10, 310 (2019).

653 77. Maini Rekdal, V., Bess, E. N., Bisanz, J. E., Turnbaugh, P. J. \& Balskus, E. P.

654 Discovery and inhibition of an interspecies gut bacterial pathway for Levodopa

655 metabolism. Science 364, eaau6323 (2019).

656 78. McAlpine, C. S. et al. Sleep modulates haematopoiesis and protects against

657 atherosclerosis. Nature (2019) doi:10.1038/s41586-019-0948-2.

658 79. Lane, J. M. et al. Genome-wide association analyses of sleep disturbance traits

659 identify new loci and highlight shared genetics with neuropsychiatric and metabolic traits.

660 Nat. Genet. 49, 274-281 (2016).

661 80. Cappuccio, F. P. et al. Meta-analysis of short sleep duration and obesity in 662 children and adults. Sleep 31, 619-26 (2008).

663 81. Cedernaes, J. et al. Acute sleep loss results in tissue-specific alterations in

664 genome-wide DNA methylation state and metabolic fuel utilization in humans. Sci. Adv.

665 4, eaar8590 (2018).

666 82. Barton, W. et al. The microbiome of professional athletes differs from that of

667 more sedentary subjects in composition and particularly at the functional metabolic level.

668 Gut gutjnl-2016-313627 (2017) doi:10.1136/gutjnl-2016-313627.

669 83. Price, N. D. et al. A wellness study of 108 individuals using personal, dense,

670 dynamic data clouds. Nat. Biotechnol. 35, 747-756 (2017).

671 84. Jensen, C. S. et al. Exercise as a potential modulator of inflammation in patients

672 with Alzheimer's disease measured in cerebrospinal fluid and plasma. Exp. Gerontol. 121,

673 91-98 (2019).

674 85. da Silva, D. E. et al. High-Intensity Interval Training in Patients with Type 2

675 Diabetes Mellitus: a Systematic Review. Curr. Atheroscler. Rep. 21, 8 (2019).

676 86. Popovic, B. et al. Acute Response to Endurance Exercise Stress: Focus on

677 Catabolic/anabolic Interplay Between Cortisol, Testosterone, and Sex Hormone Binding

678 Globulin in Professional Athletes. J. Med. Biochem. 38, 6-12 (2019).

679 87. Maldonado-Gómez, M. X. et al. Stable Engraftment of Bifidobacterium longum

680 AH1206 in the Human Gut Depends on Individualized Features of the Resident

681 Microbiome. Cell Host Microbe 20, 515-526 (2016).

682 88. Pan, H. et al. A gene catalogue of the Sprague-Dawley rat gut metagenome.

683 GigaScience 7, (2018).

684 89. Pan, H. et al. A single bacterium resurrects the microbiome-immune balance to

685 protect bones from destruction in a rat model of rheumatoid arthritis. Microbiome 7, 107

686 (2019). 
687 90. Yatsunenko, T. et al. Human gut microbiome viewed across age and geography.

688 Nature 486, 222-7 (2012).

689 91. Valenzuela, J. A., Flórez, A. B., Vázquez, L., Vasek, O. M. \& Mayo, B.

690 Production of $\gamma$-aminobutyric acid (GABA) by lactic acid bacteria strains isolated from

691 traditional, starter-free dairy products made of raw milk. Benef. Microbes 10, 579-587

692 (2019).

693 92. Daisley, B. A. et al. Immobilization of cadmium and lead by Lactobacillus

694 rhamnosus GR-1 mitigates apical-to-basolateral heavy metal translocation in a Caco-2

695 model of the intestinal epithelium. Gut Microbes 1-13 (2018)

696 doi:10.1080/19490976.2018.1526581.

697 93. Han, M. et al. A novel affordable reagent for room temperature storage and

698 transport of fecal samples for metagenomic analyses. Microbiome 6, 43 (2018).

699 94. Fang, C. et al. Assessment of the cPAS-based BGISEQ-500 platform for

700 metagenomic sequencing. GigaScience 7, 1-8 (2018).

701 95. Zhang, W. et al. IMonitor: A Robust Pipeline for TCR and BCR Repertoire

702 Analysis. Genetics 201, 459-472 (2015).

703 96. Marbach, D. et al. Wisdom of crowds for robust gene network inference. Nat.

704 Methods 9, 796-804 (2012).

705 97. Louppe, G., Wehenkel, L., Sutera, A. \& Geurts, P. Understanding variable

706 importances in forests of randomized trees. in Advances in Neural Information

707 Processing Systems 26 (eds. Burges, C. J. C., Bottou, L., Welling, M., Ghahramani, Z. \&

708 Weinberger, K. Q.) 431-439 (Curran Associates, Inc., 2013).

709 98. Morgan, X. C. et al. Dysfunction of the intestinal microbiome in inflammatory

710 bowel disease and treatment. Genome Biol. 13, R79 (2012). 


\section{Online Methods :}

\section{Study Cohort}

727 As part of 4D-SZ, all the $>2000$ volunteers for the first cohort were recruited

728 between May 2017 and July 2017 during a physical examination. The 1400 volunteers for

729 the second cohort were also recruited in 2017, with no overlaps. The samples in each

730 omics are shown in Supplementary Table 1c. Baseline characteristics of the cohort are

731 shown in Supplementary Table 1b, $1 \mathrm{~d}$.

732 The study was approved by the Institutional Review Boards (IRB) at BGI-Shenzhen, 733 and all participants provided written informed consent at enrolment.

\section{Demographic Data Collection}

736 The lifestyle questionnaire contained 56 entries involving age, marital status, disease

737 history of the volunteer and his/her family, eating and exercise habits (Supplementary

738 Table 1b, 1d). The psychological questionnaire contained 18 entries for the evaluation of

739 irritability, dizziness, frustration, fear, appetite, self-confidence, resilience

740 (Supplementary Table 1b). 


\section{Samples Collection}

743 Fecal samples were self-collected by volunteers, using a kit containing a room

744 temperature stabilizing reagent to preserve the metagenome ${ }^{93}$. The samples were frozen

745 on the same day. The overnight fasting blood samples were drawn from a cubital vein of

746 volunteers by the doctors.

747

748 DNA extraction and metagenomics shotgun sequencing

749 DNA extraction of the stored fecal samples within the next few months was

750 performed as previously described (Qin et al., 2012). Metagenomic sequencing was done

751 on the BGISEQ-500 platform (100bp of singled-end reads for fecal samples and four

752 libraries were constructed for each lane) ${ }^{94}$.

753

\section{Amino Acid Measurements}

$755 \quad 40 \mu 1$ plasma was deproteinized with $20 \mu 110 \%$ (w/v) sulfosalicylic acid (Sigma)

756 containing internal standards, then $120 \mu 1$ aqueous solution was added. After centrifuged,

757 the supernatant was used for analysis. The analysis was performed by ultra high pressure

758 liquid chromatography (UHPLC) coupled to an AB Sciex Qtrap 5500 mass spectrometry

759 (AB Sciex, US) with the electrospray ionization (ESI) source in positive ion mode. A

760 Waters ACQUITY UPLC HSS T3 column $(1.8 \mu \mathrm{m}, 2.1 \times 100 \mathrm{~mm})$ was used for amino

761 compound separation with a flow rate at $0.5 \mathrm{ml} / \mathrm{min}$ and column temperature of $55^{\circ} \mathrm{C}$.

762 The mobile phases were (A) water containing $0.05 \%$ and $0.1 \%$ formic acid (v/v), (B)

763 acetonitrile containing $0.05 \%$ and $0.1 \%$ formic acid $(\mathrm{v} / \mathrm{v})$. The gradient elution was $2 \% \mathrm{~B}$

764 kept for $0.5 \mathrm{~min}$, then changed linearly to $10 \% \mathrm{~B}$ during $1 \mathrm{~min}$, continued up to $35 \% \mathrm{~B}$ in 
$7652 \mathrm{~min}$, increased to $95 \% \mathrm{~B}$ in $0.1 \mathrm{~min}$ and maintained for $1.4 \mathrm{~min}$. Multiple Reaction

766 Monitoring (MRM) was used to monitor all amino compounds. The mass parameters

767 were as follows, Curtain gas flow $35 \mathrm{~L} / \mathrm{min}$, Collision Gas (CAD) was medium, Ion

768 Source Gas 1 (GS 1) flow 60 1/min, Ion Source Gas 2 (GS 2) flow 60 1/min, IonSpray

769 Voltage (IS) $5500 \mathrm{~V}$, temperature $600{ }^{\circ} \mathrm{C}$. All amino compound standards were purchased

770 from sigma and Toronto research chemical (TRC).

771

\section{Hormone Measurements}

$773250 \mu 1$ plasma was diluted with $205 \mu 1$ aqueous solution, For SPE experiments, HLB

774 (Waters, USA) was activated with $1.0 \mathrm{ml}$ of dichloromethane, acetonitrile, methanol,

775 respectively and was equilibrated with $1.0 \mathrm{ml}$ of water. The pretreated plasma sample was

776 loaded onto the cartridge and was extracted using gravity. Clean up was accomplished by

777 washing the cartridges with $1.0 \mathrm{ml}$ of $25 \%$ methanol in water. After drying under

778 vacuum, samples on the cartridges were eluted with $1.0 \mathrm{ml}$ of dichloromethane. The

779 eluted extract was dried under nitrogen and the residual was dissolved with $25 \%$

780 methanol in water and was transferred to an autosampler vial prior to LC-MS/MS

781 analysis. The analysis was performed by UHPLC coupled to an AB Sciex Qtrap 5500

782 mass spectrometry (AB Sciex, US) with the atmospheric pressure chemical ionization

783 (APCI) source in positive ion mode. A Phenomone Kinetex C18 column $(2.6 \mu \mathrm{m}, 2.1 \times$

$78450 \mathrm{~mm}$ ) was used for steroid hormone separation with a flow rate at $0.8 \mathrm{ml} / \mathrm{min}$ and

785 column temperature of $55^{\circ} \mathrm{C}$. The mobile phases were $(\mathrm{A})$ water containing $1 \mathrm{mM}$

786 Ammonium acetate, (B) Methanol containing 1mM Ammonium acetate. The gradient

787 elution was $25 \% \mathrm{~B}$ kept for $0.9 \mathrm{~min}$, then changed linearly to $40 \% \mathrm{~B}$ during $0.9 \mathrm{~min}$, 
788 continued up to $70 \% \mathrm{~B}$ in $2 \mathrm{~min}$, increased to $95 \% \mathrm{~B}$ in $0.1 \mathrm{~min}$ and maintained for 1.6

789 min. Multiple Reaction Monitoring (MRM) was used to monitor all steroid hormone

790 compounds. The mass parameters were as follows, Curtain gas flow 35 1/min, Collision

791 Gas (CAD) was medium, Ion Source Gas 1 (GS 1) flow 60 1/min, Ion Source Gas 2 (GS

792 2) flow 60 1/min, Nebulizer Current (NC) 5, temperature $500{ }^{\circ} \mathrm{C}$. All steroid hormone

793 profiling compound standards were purchased from sigma, Toronto research chemical

794 (TRC), Cerilliant and DR. Ehrenstorfer.

795

796 Trace element Measurements

$797200 \mu \mathrm{l}$ of whole blood were transferred into a $15 \mathrm{~mL}$ polyethylene tube and diluted 798 1:25 with a diluent solution consisting of $0.1 \%(\mathrm{v} / \mathrm{v})$ Triton X-100, $0.1 \%(\mathrm{v} / \mathrm{v})$

799 HNO3,2mg/L AU, and internal standards $(20 \mu \mathrm{g} / \mathrm{L})$. The mixture was sonicated for

$80010 \mathrm{~min}$ before ICP-MS analysis. Multi-element determination was performed on an

801 Agilent 7700x ICP-MS (Agilent Technologies, Tokyo, Japan) equipped with an octupole

802 reaction system (ORS) collision/reaction cell technology to minimize spectral

803 interferences. The continuous sample introduction system consisted of an autosampler, a

804 quartz torch with a 2.5-mmdiameter injector with a Shield Torch system, a Scott double-

805 pass spray chamber and nickel cones (Agilent Technologies, Tokyo, Japan). A glass

806 concentric MicroMist nebuliser (Agilent Technologies, Tokyo, Japan) was used for the

807 analysis of diluted samples.

808

809 Water-soluble Vitamins Measurements 
$810200 \mu 1$ plasma were deproteinized with $600 \mu 1$ methanol (Merck), water, acetic acid

811 (9:1:0.1) containing internal standards, thiamine-(4-methyl-13C-thiazol-5-yl-13C3)

812 hydrochloride (Sigma-Aldrich), levomefolic acid-13C, d3, riboflavin-13C,15N2, 4-

813 pyridoxic acid-d3 and pantothenic acid-13C3,15N hemi calcium salt (Toronto Research

814 Chemicals). $500 \mu 1$ supernatant were dried by nitrogen flow. $60 \mu 1$ water were added to

815 the residues, vortexed, the mixture was centrifuged and the supernatant was for analysis.

816 The analysis was performed by UPLC coupled to a Waters Xevo TQ-S Triple Quad mass

817 spectrometry (Waters, USA) with the electrospray ionization (ESI) source in positive ion

818 mode. A Waters ACQUITY UPLC HSS T3 column $(1.7 \mu \mathrm{m}, 2.1 \times 50 \mathrm{~mm})$ was used for

819 water-soluble vitamins separation with a flow rate at $0.45 \mathrm{ml} / \mathrm{min}$ and column

820 temperature of $45^{\circ} \mathrm{C}$. The mobile phases were (A) $0.1 \%$ formic acid in water, (B) $0.1 \%$

821 formic acid in methanol. The following elution gradient was used: $0-1 \mathrm{~min}, 99.0 \%-99.0 \%$

822 A; 1-1.5 min, 99.0\% A-97.0\% A; 1.5-2 min, 97.0\% A-70.0\% A,2-3.5 min, 70\% A-

$82330 \% \mathrm{~A} ; 3.5-4.0 \mathrm{~min}, 30 \% \mathrm{~A}-10.0 \% \mathrm{~A} ; 4.0-4.8 \mathrm{~min}, 10 \% \mathrm{~A}-10.0 \% \mathrm{~A} ; 4.9-6.0 \mathrm{~min}$,

824 99.0\% A-99.0\%A. Multiple Reaction Monitoring (MRM) was used to monitor all water-

825 soluble vitamins. The mass parameters were as follows, the capillary voltages of 3000V

826 and source temperature of $150^{\circ} \mathrm{C}$ were adopted. The desolvation temperature was $500^{\circ} \mathrm{C}$.

827 The collision gas flow was set at $0.10 \mathrm{ml} / \mathrm{min}$. The cone gas and desolvation gas flow

828 were 150 l/h and 1000 l/h, respectively. All water-soluble vitamins standards were

829 purchased from Sigma-Aldrich (USA).

830

831 Fat-soluble Vitamins Measurements 
$832 \quad 250 \mu 1$ plasma were deproteinized with $1000 \mu 1$ methanol and acetonitrile, $(\mathrm{v} / \mathrm{v}, 1: 1)$

833 (Fisher Chemical) containing internal standards, all-trans-Retinol-d5, 25-

834 HydroxyVitamin-D2-d6, 25-HydroxyVitamin-D3-d6, vitamin K1-d7, $\alpha$-Tocopherol-d6

835 (Toronto Research Chemicals). $900 \mu 1$ supernatant were dried by nitrogen flow. $80 \mu 1$

$83680 \%$ acetonitrile were added to the residues, vortexed, the mixture was centrifuged, and

837 the supernatant was used for analysis. The analysis was performed by UPLC coupled to

838 an AB Sciex Qtrap 4500 mass spectrometry (AB Sciex, USA) with the atmospheric

839 pressure chemical ionization (APCI) source in positive ion mode. A Waters ACQUITY

840 UPLC BEH C18 column $(1.7 \mu \mathrm{m}, 2.1 \times 50 \mathrm{~mm})$ was used for fat-soluble vitamins

841 separation with a flow rate at $0.50 \mathrm{ml} / \mathrm{min}$ and column temperature of $45^{\circ} \mathrm{C}$. The mobile

842 phases were (A) $0.1 \%$ formic acid in water, (B) $0.1 \%$ formic acid in acetonitrile. The

843 following elution gradient was used: 0-0.5 min,60.0\%-60.0\% A; 0.5-1.5 min, 60.0\% A-

$844 \quad 20.0 \%$ A; $1.5-2.5 \mathrm{~min}, 20.0 \%$ A-0\% A,2.5-4.5 min, 0\% A-0\% A; 4.5-4.6 min, 0\% A-

845 60.0\% A; 4.6-5.0 min, 60.0\%A-60.0\%A. Multiple Reaction Monitoring (MRM) was

846 used to monitor all fat-soluble vitamins. The mass parameters were as follows, Curtain

847 gas flow 30 1/min, Collision Gas (CAD) was medium, Ion Source Gas 1 (GS 1) flow 40

848 1/min, Ion Source Gas 2 (GS 2) flow 50 1/min, Nebulizer Current (NC) 5, temperature 400

$849{ }^{\circ} \mathrm{C}$. All fat-soluble vitamins standards were purchased from Sigma-Aldrich (USA),

850 Toronto research chemical (TRC).

851

852 Immune indices Measurements

$85310 \mathrm{ml}$ whole blood was centrifuged at 3,000 r/min for $10 \mathrm{~min}$, then $165 \mu \mathrm{l}$ buffy coat

854 were obtained to extract DNA using MagPure Buffy Coat DNA Midi KF Kit (Magen, 
855 China). The DNA was sequenced on the BGISEQ- 500 platform using 200 bp singled-

856 end reads. The data processing was performed using Immune IMonitor ${ }^{95}$. VJ Gene use

857 diversity is shannon index of VJ gene usage profile. Immune cell diversity is Shannon

858 index of CDR3. Immune cell species result is unique CDR3 number. Immunity

859 uniformity is CDR3 pielou index. Score of above index is the sample rank in population.

\section{Medical Parameters}

862 All the volunteers were recruited during the physical examination. The medical test

863 including blood tests, urinalysis, routine examination of cervical secretion. All the

864 medical parameters were measured by the physical examination center and shown in

865 Supplementary Table 1b, 1d.

866

\section{$867 \quad$ Facial Skin feature}

868 The volunteer's frontal face without makeup was photographed by VISIA-CRTM

869 imaging system (Canfield Scientific, Fairfield, NJ, USA) equipped with chin supports

870 and forehead clamps that fix the face during the photographing process and maintain a

871 fixed distance between the volunteers and the camera at all times. Eight indices were

872 obtained including spots, pores, wrinkles, texture, UV spots, porphyrins, brown spots and

873 red area from the cheek and forehead, respectively (Supplementary Table 1b). The

874 percentile of index was calculated based on the index value ranked in the age-matched

875 database, the higher the better (Supplementary Table 1b).

876

\section{Physical fitness test}


$878 \quad 8$ kinds of tests were performed to evaluate volunteers' physical fitness condition

879 (Supplementary Table 1b). Vital capacity was measured by HK6800-FH (Hengkangjiaye,

880 China). Eye-closed and single-legged standing was measured by HK6800-ZL. Choice

881 reaction time was measured by HK6800-FY. Grip strength was measured by HK6800-

882 WL. Sit and reach was measured by HK6800-TQ. Sit-ups was measured by HK6800-YW.

883 Step index was measured by HK6800-TJ. Vertical jump was measured by HK6800-ZT.

884 We got a measure value from each test. Then each measure value score was assigned 1

885 through 5 based on its corresponding age-matched national standards (Supplementary

886 Table 4). Both the direct measurements and the scores were used for analyses

887 (Supplementary Table 2, Supplementary Table 3).

888

889 Quality control, taxonomic annotation and abundance calculation

890 The sequencing reads were quality-controlled as described previously ${ }^{94}$. Taxonomic

891 assignment of the high-quality fecal metagenomic data was performed using the reference

892 gene catalog comprising 9,879,896 gene ${ }^{12}$. Taxonomy of the fecal MGSs/MLGs were

893 then determined from their constituent genes, as previously described ${ }^{1,13,14,35}$.

\section{The factors in each type of omics predicted by other type omics}

896 The factors in each type of omics were regressed against the relative abundances of

897 mgs profile (found in at least $10 \%$ of the samples) in the fecal samples using default

898 parameters in the RFCV function from randomForest package in R. Dichotomous

899 variables (such as gender) and unordered categorical variable (such as region) were re-

900 coding into dummy variables. Frequency items such as yogurt eating habit were assigned 
901 integers. RFCV R defined as spearman's correlation between measured value and 5-fold

902 cross-validation predicted value was calculated, and then rank the top 5 predictable

903 factors in each omics type. The same prediction process was done between any two types

904 of omics. Then ggplot2 package in $\mathrm{R}$ was used to boxplot predict power of target omics

905 factors by all kinds of other predictor omics (Fig. 2b). 75\% quantile RFCV R between

906 any two types omics (from $a$ to $b$ and from $b$ to $a$ ) was used to construct the bi-direction

907 global omics correlation network using CytoScape (Fig. 2a). R pheatmap and barplot was

908 used to make heatmap plot for some representative factors (Fig. 2c, Supplementary Fig.

909 1).

910

\section{Adjusting for potential confounders}

912 Associations between gut microbiome MGSs, functional modules, Shannon

913 diversity, and variance explained and other omics data were all adjusted for factors that

914 probably influence the gut microbiome, including gender, age, BMI, health products

915 (amino acid, vitamin, calcium), antivirus, antibiotics, drugs (currently using

916 antihypertensive drugs, hyperglycemic drugs, lipid lowering drugs), days since last

917 menstrual bleeding, pregnant, lactation, bowel problem (defecation). Besides the above

918 basic set of confounders, we also show the results adjusting for more potential

919 confounders including dietary (dietary taste spicy, sweet, salty, oil, or light, high sugar

920 and high-fat diet habit, fruit and vegetable intake, favors fat meat), exercise (exercise

921 frequency, exercise intensity, average time per exercise), drinking, smoking and Bristol's 922 stool score. 


\section{Benjamini-Hochberg multiple hypothesis testing correction}

925 The multiple hypothesis testing Benjamini-Hochberg corrections are done for one

926 source target omics pair each time for Fig. 4-6, except immune index and gut microbe

927 pair which $\mathrm{BH}$-adjust was done on one immune index each time. We show two versions

928 of Benjamini-Hochberg correction for Shannon and other omics in Supplementary Table

929 2a. One of the BH adjust was done within one omics each time. Another adjust was done

930 overall on all omics.

932 Robust association network construction between any two omics data type including

933 fecal microbial MGSs

934 An rank average method ${ }^{96}$ was used to combine the results of multiple inference

935 methods to make a robust omics association network. We combined two non-linear

936 models, one-to-many randomforest and one-to-one partial spearman's correlation, to test

937 the association between factor from any two types omics.

$938 \quad$ Step 1: Data preprocessing.

939 Dichotomous variables (such as gender) and unordered categorical variable (such as

940 region) were re-coding into dummy variables. Frequency items such as yogurt eating

941 habit were assigned integers. We removed variables following these rules: (i) The

942 microbial species less than $10 \%$ in all the samples. (ii) Near zero variance. (iii) With

943 more than $70 \%$ missing value. Missing values were filled with median. Outliers were

944 defined as outside of the $95 \%$ quartiles and outliers samples are removed.

945 Step 2: Computation of associations using multiple inference methods. 
946 For each factor in one omics, we did regression using RFCV function with default

947 parameter based on all factors in one other omics and calculated RFCV R. ${ }^{97} .5$-fold

948 average variable importance was output for step3. Partial spearman's correlation (ppcor R

949 package) between factors from any two types of omics were also output. Potential

950 confounders were considered as described above. We also show generalized linear model

951 results from MaAslin $\mathrm{R}$ package ${ }^{98}$ ) with default parameters after adjusting above

952 confounders.

$953 \quad$ Step 3: Robust networks construction.

954 To get the robust and strongest association between factors from any two type omics,

955 in other words, to filter predictor factors and target factors, we did it in two steps. First to

956 choose the target factors, we just kept the top 20 target factors with highest RFCV R.

957 Then to choose predictor factors for every selected target factor, we kept predictor factors

958 with top 30 average ranks and retained edges with partial spearman's correlation BH-

959 adjusted pvalue $<0.05$. The average rank was computed as sum of the ranks across the

960 RFCV importance and absolute partial spearman rho. For example, metabolites as target

961 and gut microbe as source. We regressed gut microbes against the metabolites and

962 compute the 5-fold cross validation predict power (RFCV R) for each metabolites and

963 partial spearman correlation. 20 metabolites with highest RFCV were kept. For each of

964 the 20 select metabolites such as VA, average ranks across RFCV and partial spearman

965 were done. Gut microbe biomarker for VA was found with average rank top $30^{\text {th }}$ and

966 passed the partial spearman $\mathrm{BH}$-adjusted pvalue $<0.05$.

$967 \quad$ Step 4: Network visualization. 
968 For each target factor, top 5-10 average ranks source factor in each source omics

969 type were selected as representative factors to make barplots using ggplot2 package (Fig.

970 6). The pheatmap package was used to plot the common representative factors that could

971 be strongest predicted by multiple omics data type (Fig. 2c). All the source-target factors

972 pair RFCVR (a as source, $b$ as target and b as source, a as target) was boxplot (Fig. 2b)

973 using ggplot2. The ComplexHeatmap package in $\mathrm{R}$ was used to plot omics triadic relation

974 (Fig. 4-6). CytoScape was also used to visualize the global omics network (Fig. 2a).

976 Microbial metabolic syndrome risk index validation in cardiometabolic cohort.

977 Using multi-omics analyses method described above after controlling for the

978 potential confounders above, we picked up 80 MGSs that significantly correlated with

979 one of the eight cardiometabolic risk factors (waist Hip Ratio, BMI, triglyceride

980 (mmol/L), High-Density-Lipoprotein (mmol/L), serum Uric Acid ( $\mu$ mol/L), $\gamma$-glutamyl

981 transpeptidase (U/L), serum alanine aminotransferase(U/L), fasting blood glucose

$982(\mathrm{mmol} / \mathrm{L}))($ Supplementary Fig. 5, 6). And they are link to the BCAA metabolites (valine

983 / leucine / alanine), tryptophan, glutamic acid ( $<<0.1$, Supplementary Table 3a). For the

984 published disease studies from China, all the MGSs abundances were derived from

985 metagenomic shotgun data, while the 8 clinical measurements could be missing, e.g. liver

986 cirrhosis and Crohn's disease only had BMI available ${ }^{21,31,32,34,35}$ (Supplementary Fig. 5,

987 6). The microbial metabolic syndrome risk index is similar with the T2D index (Qin et al,

988 2012). For each individual validation sample, the microbial metabolic syndrome risk

989 index of sample $j$ that denoted by $M M S R j$ was computed by the formula below:

$$
P R_{i j}=\operatorname{Count}\left(A_{i j}>R_{i}\right) / N
$$




$$
\begin{gathered}
J^{B}=\sum_{i \in B} P R_{i j} \\
J^{G}=\sum_{i \in G} P R_{i j} \\
M M S R_{j}=\frac{J^{B}}{|B|}-\frac{J^{G}}{|G|}
\end{gathered}
$$

990 Where $A i j$ is a scalar represents the relative abundance of MGS $i$ in validation

991 sample $j . R i$ is a vector represents the relative abundance of MGS $i$ of all samples in this

992 cohort which served as healthy reference. $N$ is the sample size of this cohort that is 2183.

993 Percentile rank PR $i j$ is the percentage of test sample $j$ 's MGS $i$ relative abundance in its

994 reference cohort frequency distribution that are equal to or lower than it. $B$ is 12 out of 80

995 MGS that were positively correlated with BMI and Triglyceride. $G$ is 68 out of 80 MGS

996 that were negatively correlated with BMI and Triglyceride. And $|B|$ and $|G|$ are the sizes

997 of these two sets. We used percentile rank instead of relative abundance to avoid that the

998 index was influenced too much by the dominant species.

1000 Figure legends:

1001 Fig. 1 | Overview of the multi-omic cohorts. Diagram for features available from the 1002 main cohort of 2,183 individuals and validation cohort of 1,404 volunteers. Details are 1003 available in Supplementary Table 1.

1004 Fig. 2 | Overview of the interrelationship between omics in the main cohort. a,

1005 Global association strength between omics datasets. Each arrow is a 5-fold cross-

1006 validation random forest (RFCV) prediction. The direction of the arrow indicated the 
1007 direction of prediction, used the source omics dataset to predict the target dataset. The

1008 darkness and size of the arrow lines indicated 75\% quantile of spearman's correlation

1009 between measured value and 5-fold cross-validation RFCV predicted value (RFCV R). b,

1010 Detailed predict power of source omics for each target omics. Tick label in $\mathrm{x}$-axis is

1011 target omics. Title in top is source omics. Each node in box is a target factor. The color of

1012 the node and box line indicated the target omics data type. Y-axis is the target factor

1013 RFCV R predicted from source omics. c, Common representative factors that could be

1014 strongest predicted by multiple omics data type. Y-axis tick label is source omics. Title is

1015 target omics. X-axis tick label is common representative factors (target factors). The cell

1016 color in heat map indicated the RFCV R using the omics data in y-axis to predict each

1017 factor in $\mathrm{x}$-axis.

1018 Fig. 3 | Factors associated with gut microbiome in both cohorts. Top 45 factors with

1019 RFCV R > 0.1 in each type of omics that are predicted by gut microbiome. Factors with

$1020 \mathrm{R} \leq 0.1$ in main cohort are not shown. The length of the bar indicated the rank RFCV R

1021 using all samples and the color indicated the rank of max of RFCV R using male or

1022 female samples only, the darker the greater. Due to missing medical data in the validation

1023 cohort (Fig. 1, Supplementary Table 1), only red blood cell count can be validated.

1024 Fig. 4 | Association map of the four-tiered analyses integrating the metabolites,

1025 clinical indices, life style and the fecal microbiome. The color of heat map show the

1026 partial spearman correlation adjusted for factors that probably influence the gut

1027 microbiome, as shown in Supplementary Fig. 3. BH-adjusted p-value is denoted: +, q-

1028 value $<0.1 ; *$, q-value $<0.05 ; * *$, q-value $<0.01$ 


\section{Fig. 5 | Gut microbiome associated with physical fitness and exercise in the main}

1030 cohort. The color of heat map shows the partial spearman correlation adjusted factors

1031 that probably influence the gut microbiome, as shown in Supplementary Fig. 3. BH

1032 adjusted $\mathrm{p}$-value is denoted: + , q-value $<0.1 ; *$, q-value $<0.05$; **, q-value $<0.01$

1033 Fig. 6 | Influence of yogurt and milk intake on omics in the main cohort. a-e, The top

10345 factor in each omics data associated with yogurt, milk intake habit and the

1035 Streptococcus thermophiles, Bifidobacterium animalis and Clostridium bolteae

1036 abundance. The length of the bars represents partial Spearman's correlation coefficient

1037 adjusted for factors that probably influence the gut microbiome, as shown in

1038 Supplementary Fig. 3. BH adjusted p-value is denoted: +, q-value<0.1; *, q-value $<0.05$;

$1039 * *$, q-value $<0.01 ; * * *$, q-value $<0.001 ; * * * *$, q-value $<0.0001$. f, Fecal relative abundance

1040 of $S$. thermophilus in volunteers with increasing frequency of yogurt consumption. 
49 facial skin imaging indices (only in the main cohort)

Spots, Pores, Wrinkles, Porphyrins, Texture, UV spots, Porphyrins, Brown spots and red area from the cheek and forehead, respectively.

\section{Psychological questionnaire (18 entries)}

The evaluation of irritability, Appetite, Dizziness, Frustration, Resilience, Fear, Self-confidence...

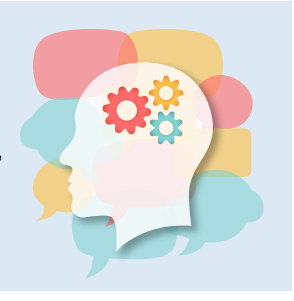

\section{Lifestyle questionnaire} (56 entries)

Age, Gender, Marital status, BMI, Smoking, Drinking, Disease history, Eating habits, Exercise habits...

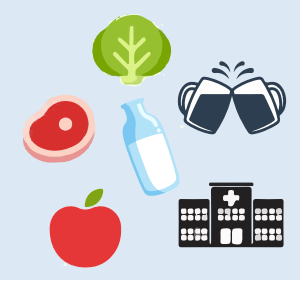

\section{Fecal microbiome}

Shotgun metagenomics (1,507 MGSs and 2,981 MLGs)

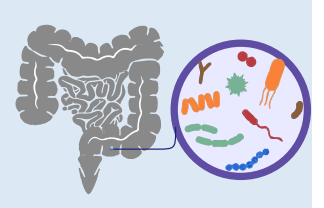

24 physical fitness data (only in the main cohort)

Vital capacity, Grip strength, Sit-ups, Choice reaction time, Sit and reach, One-leg stand with eyes closed,

Step index, Vertical jump...

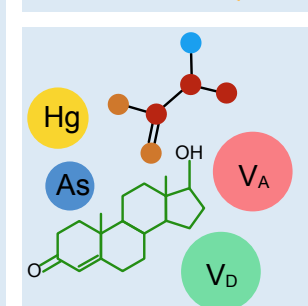

\section{4 plasma metabolites}

Amino acids (plasma)

Hormones (plasma)

Vitamins (plasma)

Trace elements (whole blood)

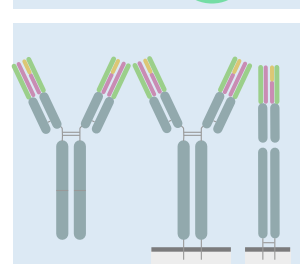

634 immune indices

(buffy coat)

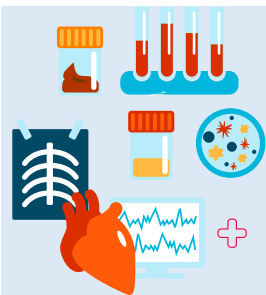

72 basic medical data (9 in validation cohort)

3,587 Healthy adults.

The main cohort: 2,183 individuals

The validation cohort: 1,404 individuals
Body measurements:

BMI, Chest circumference, Uric acid Routine blood test:

Alkaline phosphatase, Bilirubin, HDL, LDL, Globulin, Creatinine (serum) 


\section{$a$}

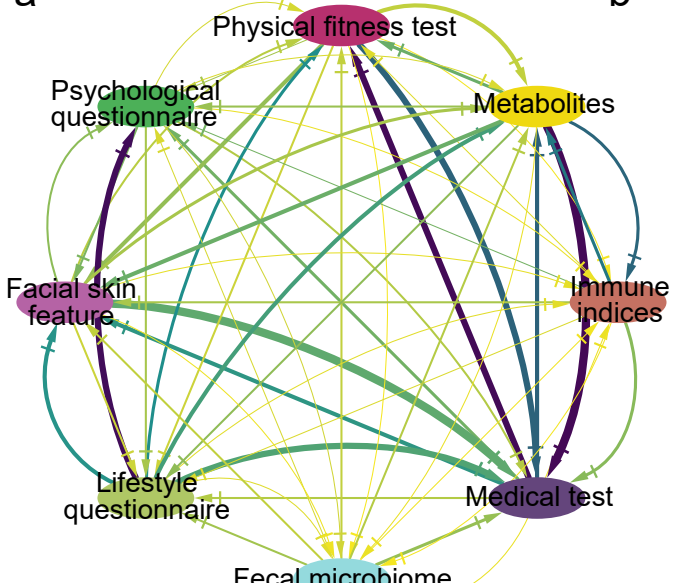

Fecal microbiome

$\begin{array}{ll}r<0.1 & -0.3<=r<0.4 \\ -0.1<=r<0.2 & =0.4<=r<0.5 \\ 0.2<=r<0.3 & r>=0.5\end{array}$
Fecal microbiome

Medical test

Immune indices

Metabolites

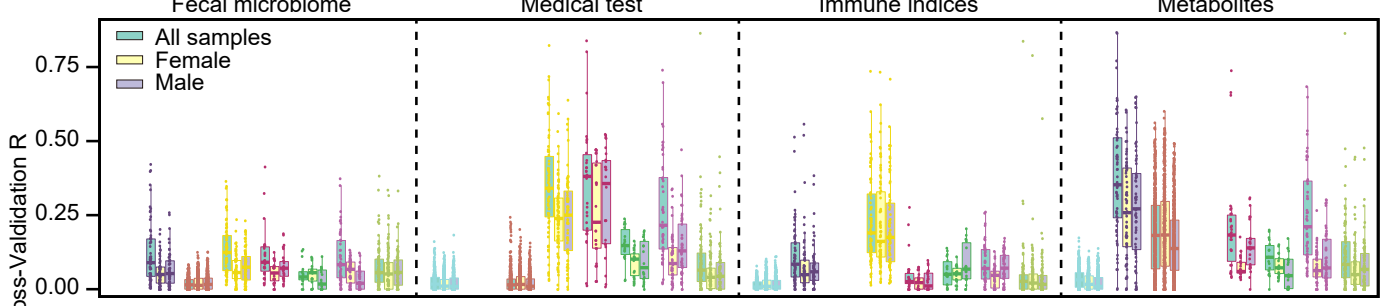

$\begin{array}{ccccc}\text { Physical fitness test } & \text { Psychological questionnaire } & \text { Facial skin feature } & \text { Lifestyle questionnaire } \\ 0 & & \end{array}$

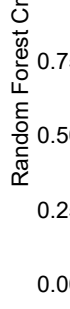

C

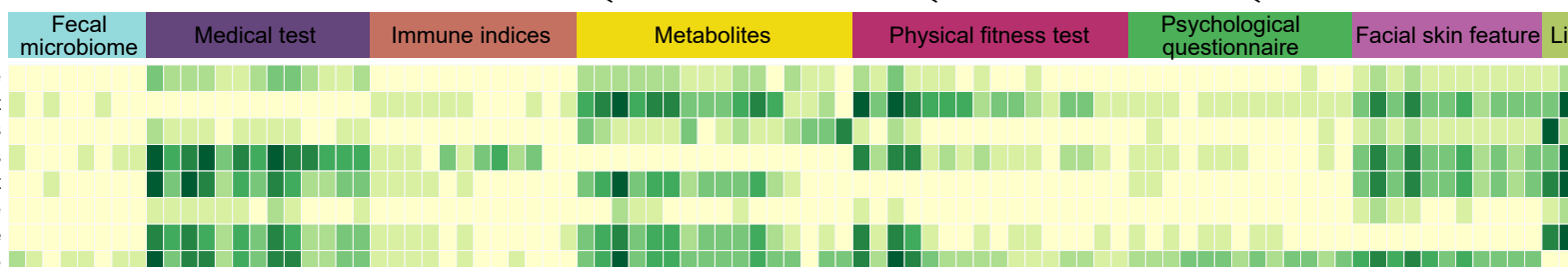

estionnaire
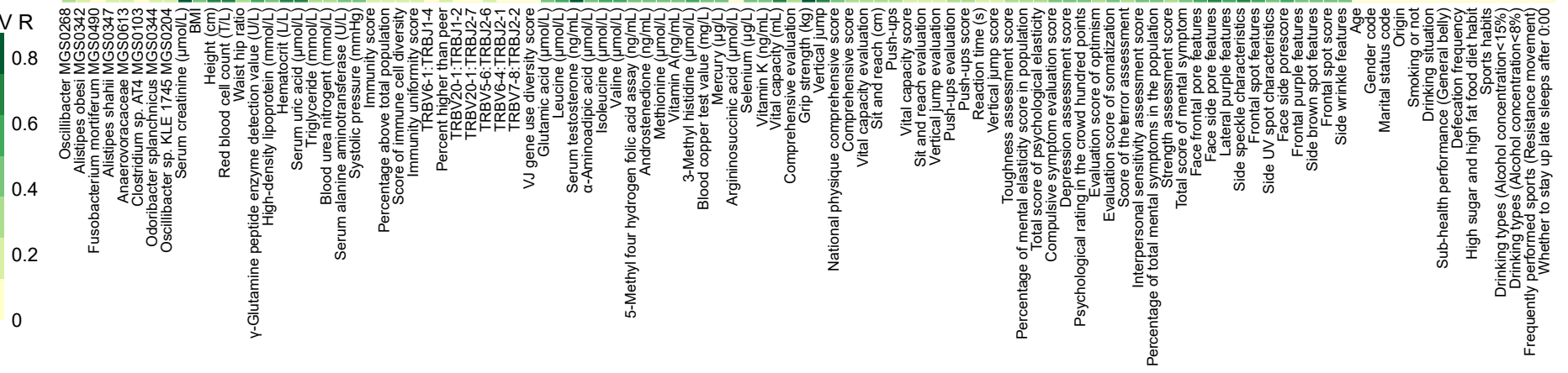


\section{Lifestyle questionnaire}

History of vaccination (Bacillus calmette guerin vaccine Sub-health performance (Cold susceptibility) story of vaccination (Polio vaccine) Nature of the Dietary taste salty drin Nearly half year drug details
Nenstrual mentiontormer) The symptoms of my irregular menstruation (Menstrual mentiontormer) Drinking types

Milk habits
Whether to stay up late sleeps after $0: 00$
Pregnancy cycle

The symptoms of my irregular menstruation (Menstrual lengthening Sub-health performance (Constipation
High sugar and high fat food diet habi

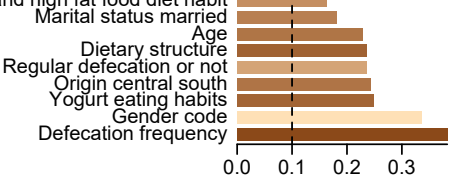

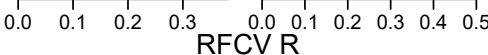
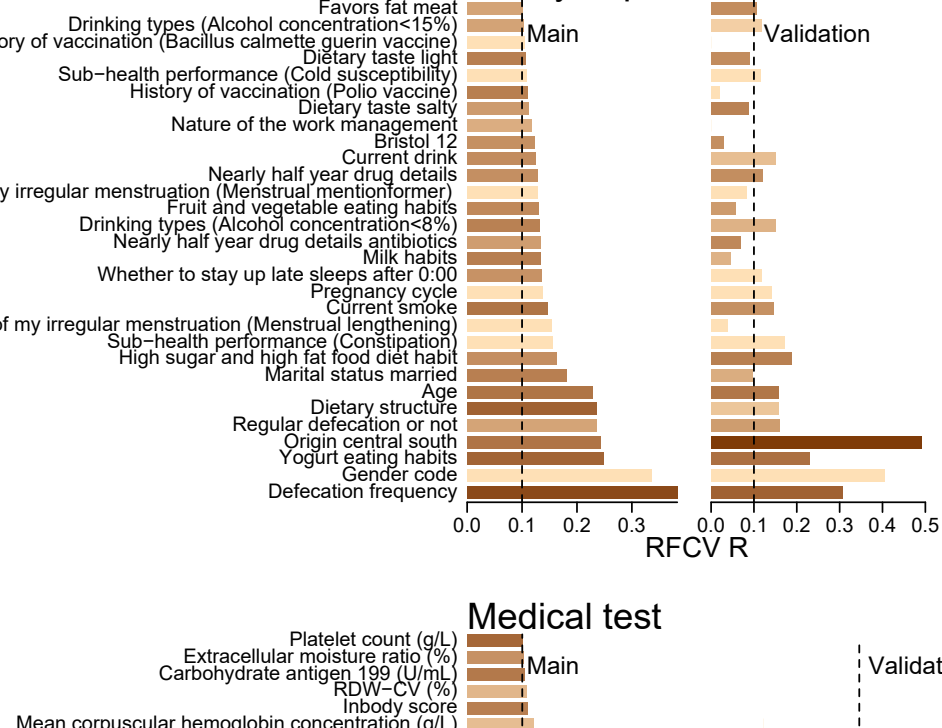

\section{Medical test} Extracellular moisture ratio $\%$
Carbohydrate antigen $199(\mathrm{U} / \mathrm{mL})$
Main Inbody scor

Mean corpuscular hemoglobin concentration (g/L) Serum alanine aminotransferase ( Y-Glutamyl transpeptidase (U RV5+SV1 amplitude (m Albumin concentration ( $\mathrm{g} / \mathrm{L}$ ABO blood group cod Diastolic pressure $(\mathrm{mmHg})$ Waist-hip ratio Blood urea nitrogent (mmol/ gh-density lipoprotein ( $\mathrm{mmol} / \mathrm{m}$

Systolic pressure
Triglyceride (mmo

Red blood cell coight (cm) serum uric acid $(\mu \mathrm{mol} / \mathrm{L}$ Serum creatinine $(\mu \mathrm{mol} / L)$
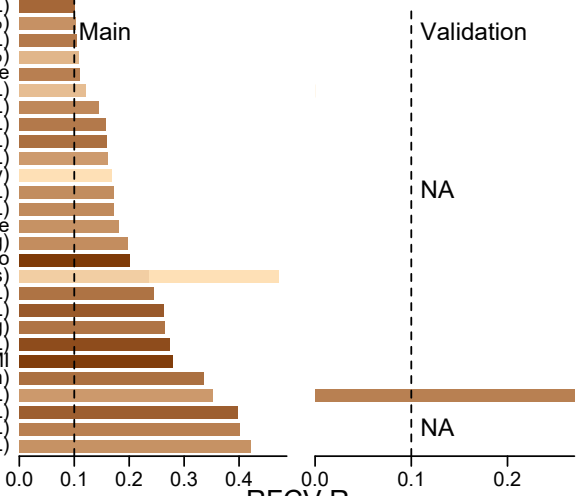
Step index
acity score $\begin{array}{lllll}0.0 & 0.1 & 0.2 & 0.3 & 0.4 \\ & & & & \\ \text { RFCV R }\end{array}$ Tyrosine ( $\mu \mathrm{mol} /$

agnesium test value $(\mathrm{mg}$ Ornithine \}, mol/

-Methyl four hydrogen folic acid assay $\mathrm{ng} / \mathrm{m}$

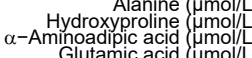$$
\text { Vitamin A }
$$
3-MethylHistidine ( ( $\mu \mathrm{mol} /$ Leucine ( $\mu \mathrm{mo}$

Serum testosterone $(\mathrm{ng} / \mathrm{m}$

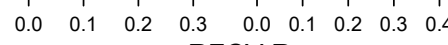
RFCV R

\section{Immune indices}

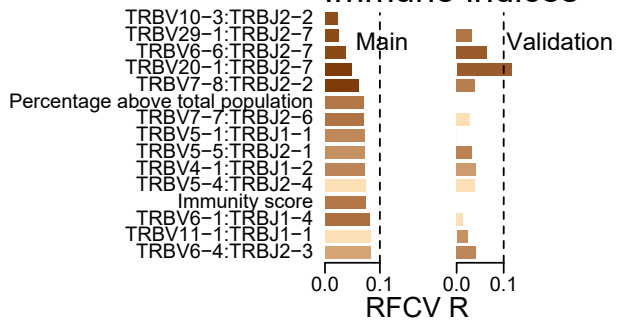

Physical fitness test

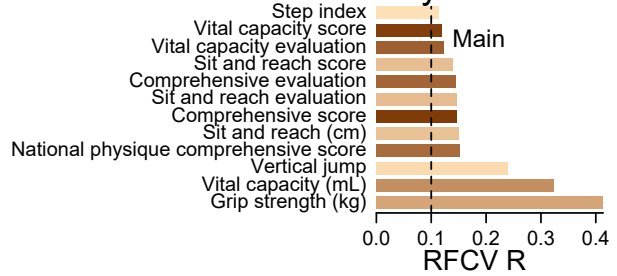

Psychological questionnaire

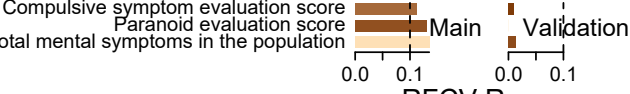

RFCV R

\section{Facial skin feature}

Frontal wrinklescore Side red zone features

Perc of front texture
Frontal purple features

Face front texture features Face frontal pore features
Frontal wrinkle features

Face side porescore

uv spot characteristics

Frontal spot features

Side brown spot features

Face side texture features

Fid Face side pore features

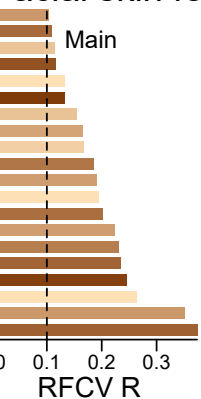

Lateral purpuricscore Frontal spotscore 


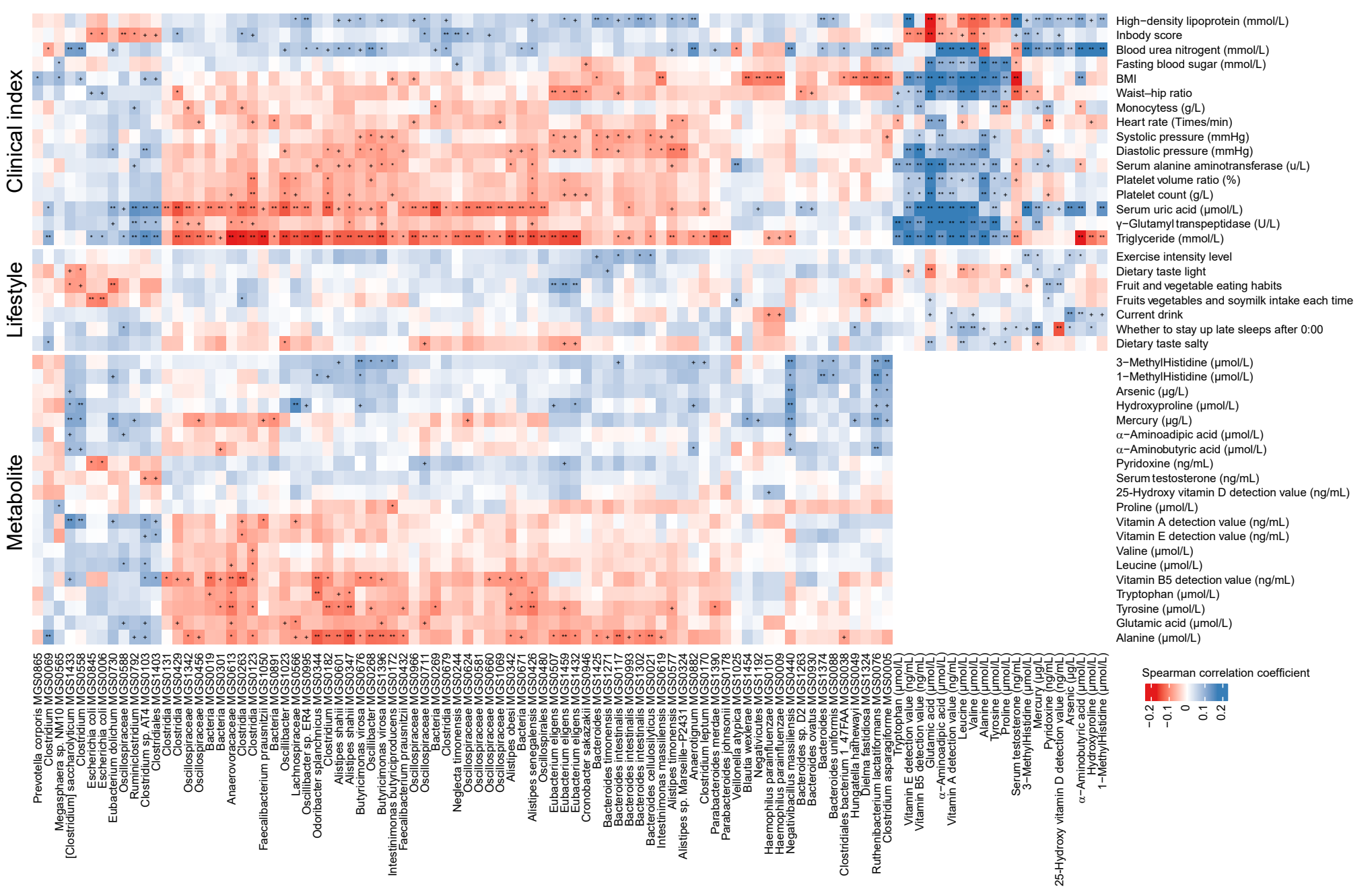




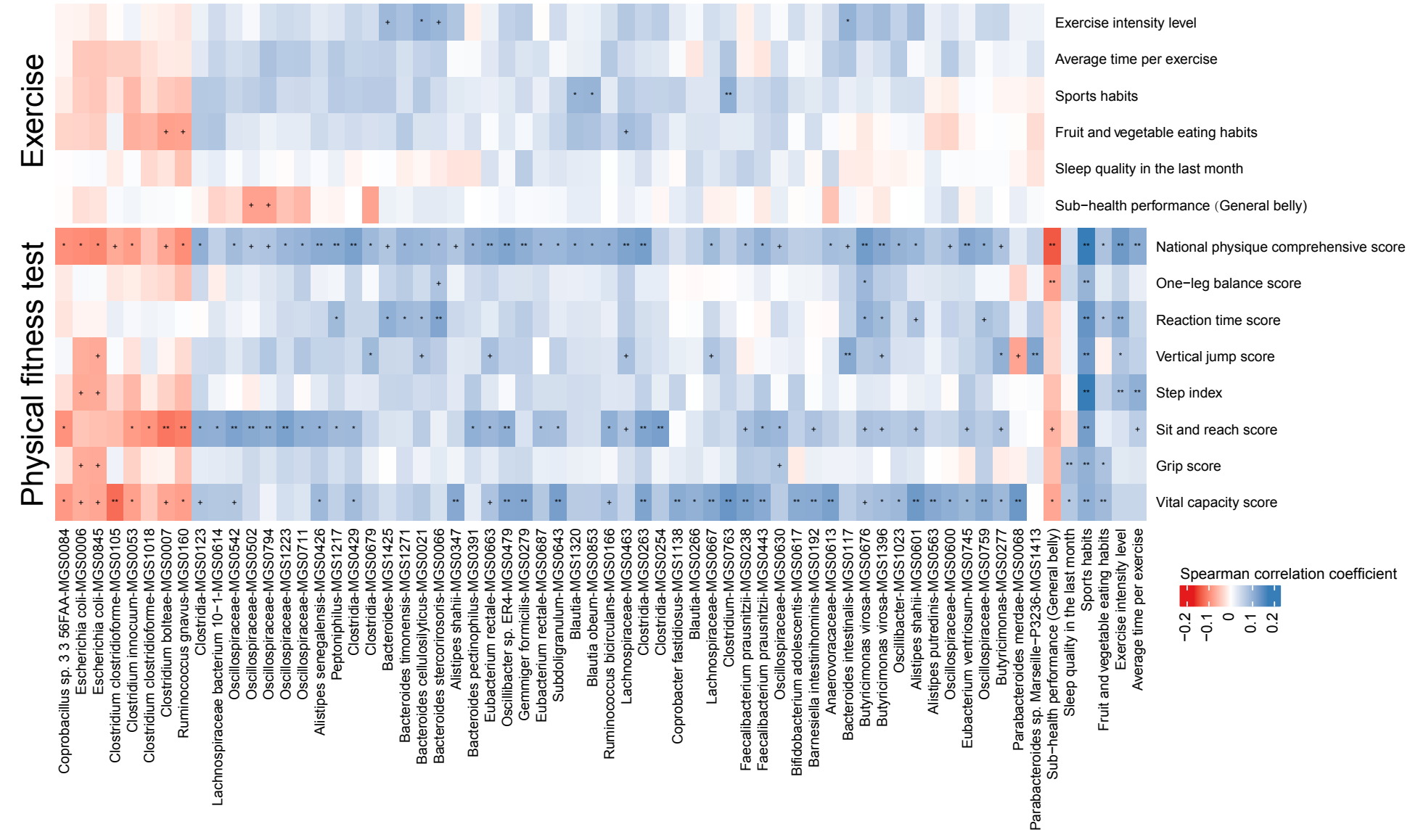


C

Streptococcus thermophilus Imd-9 MGS0852 Percentage of lateral UV stain Percent frontal spot
Percentage of front texture
Frontal spotscore

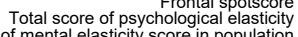
Percentage of mental elasticity score in population Psychological rating in the crowd hundred points
Percentage of total mental symptoms in the population Interpersonal sensitivity assessment score
Vital capacity evaluation
Vital capacity (mL) Vital capacity score

National physique and reach evaluation

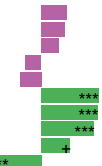

Vitamin $B 6(\mathrm{ng} / \mathrm{mL})$
Vitamin B5 $(\mathrm{ng} / \mathrm{mL})$ Vitamin $\mathrm{B} 2(\mathrm{ng} / \mathrm{mL})$
Ornithine $(u \mathrm{~mol} / \mathrm{L})$ Proline $(\mu \mathrm{mol} / \mathrm{L}$
$\mathrm{r}$ TRBV6- 3 :TRBJ2-7 TRBV7-7:TRBJ1-5 High-density lipoprotein (mmol/L) Percentage of lymphocytes $(\%$ Total neutrophils (g/

Bifidobacterium catenulatum DSM 16992 ML G24106 cterium pseudocatenulatum DSM 20438 MLG1569 Bifidobacterium catenulatum MGS1177
Bifidobacterium longum MGS0625
Bifidobacterium longum MGS1182
Blautia massiliensis MGSO228
Unclassified MLG5719
Unclassified MLG379821
Ruminococcus sp. 5 1 39 BFAA MLG118863 Blāutia wexlerae MGS0575

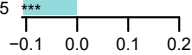
$\begin{array}{r}\text { Percentage of frontal red area } \\ \text { Frontal spotscore } \\ \text { Lateral purple features }\end{array}$
Percentage of mental elasticity score in population Percentage of mental elasticity score in population Percentage of total mental symptoms in the population Depression assessment score Vital capacity ( $\mathrm{mL}$ ) Vital capacity score National physique comprehensive score
One-leg stand (s) Lysine $(\mu \mathrm{mol} / \mathrm{L})$ Mercury $(\mu \mathrm{g}$ Hyd Vitamin $A(\mathrm{ng} / \mathrm{mL}$
$\mathrm{TRBV} 20-1: T R B J 2-4$ TRBV6-9:TRBJ2-7 TRBV24-1:TRBJ2-7 TRBV19:TRBJ2-1 High-density lipoprotein ( $\mathrm{mmol} / \mathrm{L})$ Extracellular moisture ratio $(\%)$
Serum uric acid $(\mu \mathrm{mol} / / \mathrm{L}$ Triglyceride (mmol/L

Streptococcus thermophilus CNRZZ1066 MLG55866 Streptococcus thermophilus ND03 MLG245923
Streptococcus thermophilus MGS08 Streptococcus thermophilus LMG 18311 MLG73 Bifidobacterium animalis MGS0801
Streptococcus vestibularis MGS1414
Veillonella MGS1495
Clostridium bolteae MGS0007
Clostridium bolteae ATCC BAA 613 MLG54408 Streptococcus vestibularis MGS 1414
Veillonella MGS1495
Clostridium bolteae MGSO007
Clostridium bolteae ATCC BAA 613 MLG54408

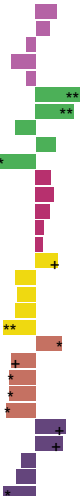
Clostridium boleae ATCC BAA 613 MLG32716
Clostridium bolteae ATCC BAA 613 MLG3 \begin{tabular}{cccc}
\hline & & 1 & \\
-0.1 & 0.0 & 0.1 & 0.2
\end{tabular}

\section{e}

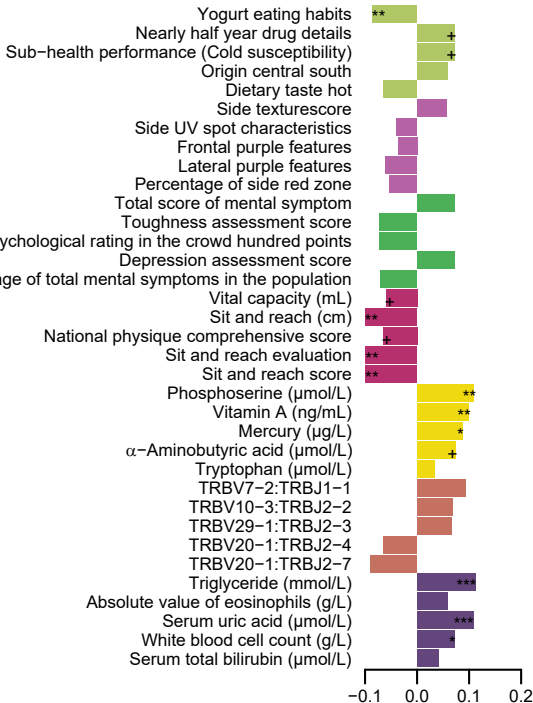

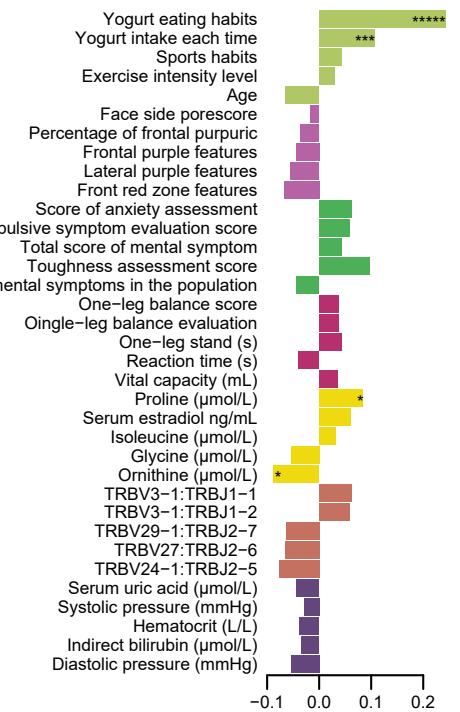

d

Bifidobacterium animalis lactis CNCM I 2494 MLG1576

Yogurt eating habit
Work statu
Date traum
Ag
Source of stress emotio
Face side porescore
Face frontal pore feature
Face side texture feature
Face side pore feature

Face side pore features

Percentage of mental elasticity score in population

Total score of mental symptom
Psychological rating in the crowd hundred points epression assessment score Percentage of total mental symptoms in the population One-leg stand (s)
Reaction time (s) Vertical jump Grip strength $(\mathrm{kg})$ Sit and reach $(\mathrm{cm})$ $\beta$-Alanine $(\mu \mathrm{mol} / \mathrm{L})$ Isoleucine $(\mu \mathrm{mol} / /)$ Tryptophan $(\mu \mathrm{mol} / /)$ omocysteine $(\mu \mathrm{mol} / \mathrm{L})$ Ornithine $(\mu \mathrm{mol} / \mathrm{L})$ TRB 0 - TRBJ2-5 TRBV20-1:TRBJ1-2 TRBV20-1:TRBJ1-1 TRBV7-9:TRBJ1-1 White blood cell count $(g / L)$ Indirect bilirubin $(\mu \mathrm{mol} / \mathrm{L})$
L Direct bilirubin $(\mu \mathrm{mol} / \mathrm{L})$ Diastolic pressure $(\mathrm{mmHg})$

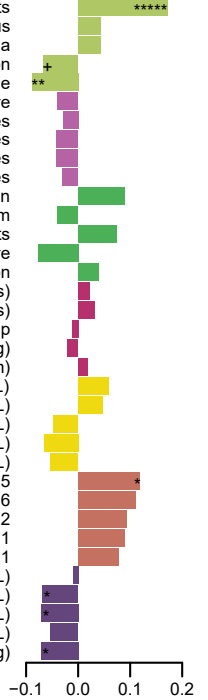

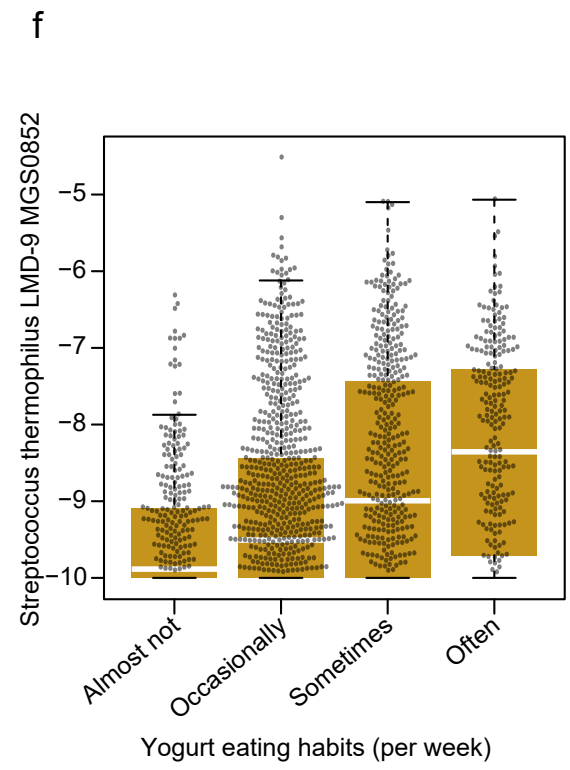

Yogurt eating habits (per week) Compusiv Percentage of total mental symptoms in the populatio 